\title{
CONFORMAL FRACTALS FOR NORMAL SUBGROUPS OF FREE GROUPS
}

\author{
JOHANNES JAERISCH
}

\begin{abstract}
We investigate subsets of a multifractal decomposition of the limit set of a conformal graph directed Markov system which is constructed from the Cayley graph of the free group $F_{d}$ with at least two generators. The subsets we consider are parametrised by a normal subgroup $N$ of $F_{d}$ and mimic the radial limit set of a Kleinian group. Our main results show that, regarding the Hausdorff dimension of these sets, various results for Kleinian groups can be generalised. Namely, under certain natural symmetry assumptions on the multifractal decomposition, we prove that, for a subset parametrised by $N$, the Hausdorff dimension is maximal if and only if $F_{d} / N$ is amenable and that the dimension is greater than half of the maximal value. We also give a criterion for amenability via the divergence of the Poincaré series of $N$. Our results are applied to the Lyapunov spectrum for normal subgroups of Kleinian groups of Schottky type.
\end{abstract}

\section{Introduction AND STATEMENT OF RESUlts}

In this paper we investigate the Hausdorff dimension of a class of conformal fractals associated with normal subgroups of free groups. Our main results show that these fractals share many interesting properties with the radial (or conical) limit set of a Kleinian group. To define the sets we consider, let $F_{d}$ denote the free group generated by $I:=\left\{g_{1}, g_{1}^{-1}, \ldots, g_{d}, g_{d}^{-1}\right\}$ with $d \geq 2$. The set of infinite reduced paths starting from the identity in the Cayley graph of $F_{d}$ with respect to $I$ is given by $\Sigma:=\left\{\tau=\left(\tau_{i}\right) \in I^{\mathbb{N}}: \tau_{i} \neq \tau_{i+1}^{-1}\right\}$. Let $\Phi$ be a conformal graph directed Markov system associated with $F_{d}=\left\langle g_{1}, \ldots, g_{d}\right\rangle$ with coding map $\pi_{\Phi}: \Sigma \rightarrow \mathbb{R}^{D}, D \geq 1$ (see Definition 3.8). Such a system $\Phi$ consists of a set of contracting conformal maps on $\mathbb{R}^{D}$ and each limit point $\pi_{\Phi}(\tau)$ of $\Phi$ is obtained by successively applying these maps according to the infinite path $\tau \in \Sigma$. We will define subsets of the limit set $\pi_{\Phi}(\Sigma)$ as follows. For a normal subgroup $N$ of $F_{d}$, the symbolic radial limit set $\Lambda_{\mathrm{r}}(N)$ of $N$ consists of those paths in $\Sigma$, for which the projection to the quotient graph $F_{d} / N$ visits some vertex infinitely often, that is,

$\Lambda_{\mathrm{r}}(N):=\left\{\tau \in \Sigma: \exists h \in F_{d}\right.$, such that $\tau_{1} \cdots \tau_{n} \in h N$ for infinitely many $\left.n \in \mathbb{N}\right\}$.

Received by the editors June 20, 2013.

2010 Mathematics Subject Classification. Primary 37C45, 30F40; Secondary 37C85, 43A07.

Key words and phrases. Kleinian groups, exponent of convergence, normal subgroups, amenability, conformal graph directed Markov systems.

The author was supported by the research fellowship JA 2145/1-1 of the German Research Foundation (DFG). 
We will investigate the sets $\pi_{\Phi}\left(\Lambda_{\mathrm{r}}(N)\right)$ and $\pi_{\Phi}\left(\Lambda_{\mathrm{r}}(N) \cap \mathcal{F}(\alpha, \Phi, \psi)\right)$ in Theorems 1.1 and 1.2, where the multifractal level sets $\mathcal{F}(\alpha, \Phi, \psi)$ are for $\alpha \in \mathbb{R}$ given by

$$
\mathcal{F}(\alpha, \Phi, \psi):=\left\{\tau \in \Sigma: \lim _{n \rightarrow \infty} \frac{\sum_{i=0}^{n-1} \psi\left(\sigma^{i}(\tau)\right)}{\sum_{i=0}^{n-1} \zeta\left(\sigma^{i}(\tau)\right)}=\alpha\right\},
$$

the potential $\psi: \Sigma \rightarrow \mathbb{R}$ is Hölder continuous, $\zeta: \Sigma \rightarrow \mathbb{R}$ is the geometric potential of $\Phi$ and $\sigma: \Sigma \rightarrow \Sigma$ refers to the left shift map. For the definition of the symbolic uniformly radial limit set $\Lambda_{\text {ur }}(N)$ we refer to Definition 3.8. We refer to Sections 2 and 3 for an introduction to symbolic thermodynamic formalism and graph directed Markov systems.

To state our first main result, we have to make further definitions. For each $n \in \mathbb{N}$, the set of admissible words of length $n$ is given by

$$
\Sigma^{n}:=\left\{\omega \in I^{n}: \omega_{i} \neq \omega_{i+1}^{-1}, 1 \leq i \leq n-1\right\} .
$$

For $\omega \in \Sigma^{n}$ and a function $f: \Sigma \rightarrow \mathbb{R}$ we define

$$
S_{\omega} f:=\sup _{\tau \in[\omega]} \sum_{i=0}^{n-1} f\left(\sigma^{i}(\tau)\right), \quad \text { where }[\omega]:=\left\{\tau \in \Sigma: \tau_{1}=\omega_{1}, \ldots, \tau_{n}=\omega_{n}\right\} .
$$

For convenience, we denote by $\varnothing$ the unique word of length zero and we set $S_{\varnothing} f:=$ 0 . We let $\Sigma^{*}:=\bigcup_{n \in \mathbb{N}} \Sigma^{n}$ and we identify $\Sigma^{*} \cup\{\varnothing\}$ with $F_{d}$. Let $N$ denote a non-trivial normal subgroup of $F_{d}$. The Poincaré series of $(N, \Phi)$ and the exponent of convergence of $(N, \Phi)$ are for $u \in \mathbb{R}$ given by

$$
P_{N}(u, \Phi):=\sum_{\omega \in N} \mathrm{e}^{u S_{\omega} \zeta} \quad \text { and } \quad \delta_{N}:=\inf \left\{u \in \mathbb{R}: P_{N}(u, \Phi)<\infty\right\} .
$$

More generally, for a Hölder continuous potential $\psi: \Sigma \rightarrow \mathbb{R}$, we introduce the free energy function of $(N, \Phi, \psi)$ which is for $\beta \in \mathbb{R}$ given by

$$
t_{N}: \mathbb{R} \rightarrow \mathbb{R}, \quad t_{N}(\beta):=\inf \left\{u \in \mathbb{R}: \sum_{\omega \in N} \mathrm{e}^{\beta S_{\omega} \psi+u S_{\omega} \zeta}<\infty\right\} .
$$

Let us also set $\delta:=\delta_{F_{d}}$ and $t:=t_{F_{d}}$. For $\beta \in \mathbb{R}$, we say that $(N, \Phi, \psi)$ is of divergence type in $\beta$ if $\sum_{\omega \in N} \mathrm{e}^{\beta S_{\omega} \psi+t_{N}(\beta) S_{\omega} \zeta}=\infty$. We say that $(N, \Phi)$ is of divergence type if $(N, \Phi, 0)$ is of divergence type in 0 , that is, $P_{N}\left(\delta_{N}, \Phi\right)=\infty$.

We need the following notions of symmetry (cf. Definition 4.1). For each $n \in \mathbb{N}$ and $\omega \in \Sigma^{n}$ we set $|\omega|:=n$ and $\omega^{-1}:=\left(\omega_{n}^{-1}, \ldots, \omega_{1}^{-1}\right)$. We say that $(N, \Phi, \psi)$ is asymptotically symmetric, if for all $\beta, u \in \mathbb{R}$ there exist $n_{0} \in \mathbb{N}$ and sequences $\left(c_{n}\right) \in\left(\mathbb{R}^{+}\right)^{\mathbb{N}}$ and $\left(N_{n}\right) \in \mathbb{N}^{\mathbb{N}}$ with $\lim _{n}\left(c_{n}\right)^{1 / n}=1$ and $\lim _{n} n^{-1} N_{n}=0$, such that for each $g \in F_{d}$ and for all $n \geq n_{0}$,

$$
\sum_{\omega \in N g:|\omega|=n} \mathrm{e}^{\beta S_{\omega} \psi+u S_{\omega} \zeta} \leq c_{n} \sum_{\omega \in N g^{-1}: n-N_{n} \leq|\omega| \leq n+N_{n}} \mathrm{e}^{\beta S_{\omega} \psi+u S_{\omega} \zeta}
$$

If $\left(c_{n}\right)$ can be chosen to be bounded, for all $\beta, u \in \mathbb{R}$, then $(N, \Phi, \psi)$ is called symmetric.

For $\beta \in \mathbb{R}$, we say that $(N, \Phi, \psi)$ is symmetric on average in $\beta$ if

$$
\sup _{g \in F_{d}} \limsup _{n \rightarrow \infty} \frac{\sum_{k=1}^{n} \sum_{\omega \in N g:|\omega|=k p} \mathrm{e}^{\beta S_{\omega} \psi+t_{N}(\beta) S_{\omega} \zeta}}{\sum_{k=1}^{n} \sum_{\omega \in N g^{-1}:|\omega|=k p} \mathrm{e}^{\beta S_{\omega} \psi+t_{N}(\beta) S_{\omega} \zeta}}<\infty
$$


where $p:=\operatorname{gcd}\left\{n \in \mathbb{N}: \exists \omega \in \Sigma^{n} \cap N\right.$ such that $\left.\omega_{n} \omega_{1} \neq \mathrm{id}\right\}$ and gcd refers to the greatest common divisor.

We say that $(N, \Phi)$ is (asymptotically) symmetric if $(N, \Phi, 0)$ is (asymptotically) symmetric, and $(N, \Phi)$ is symmetric on average if $(N, \Phi, 0)$ is symmetric on average in 0 .

Theorem 1.1. Let $\Phi$ denote a conformal graph directed Markov system associated with $F_{d}, d \geq 2$. Then the following holds for each non-trivial normal subgroup $N$ of $F_{d}$.

(1) We have

$$
\operatorname{dim}_{H}\left(\pi_{\Phi}\left(\Lambda_{\text {ur }}(N)\right)\right)=\operatorname{dim}_{H}\left(\pi_{\Phi}\left(\Lambda_{\mathrm{r}}(N)\right)\right)=\delta_{N}>0 .
$$

(2) If $F_{d} / N$ is non-amenable, then

$$
\operatorname{dim}_{H}\left(\pi_{\Phi}\left(\Lambda_{\mathrm{r}}(N)\right)\right)<\operatorname{dim}_{H}\left(\pi_{\Phi}\left(\Lambda_{\mathrm{r}}\left(F_{d}\right)\right)\right) .
$$

(3) Suppose that $(N, \Phi)$ is asymptotically symmetric.

(a) If $F_{d} / N$ is amenable, then

$$
\operatorname{dim}_{H}\left(\pi_{\Phi}\left(\Lambda_{\mathrm{r}}(N)\right)\right)=\operatorname{dim}_{H}\left(\pi_{\Phi}\left(\Lambda_{\mathrm{r}}\left(F_{d}\right)\right)\right) .
$$

(b) We have

$$
\operatorname{dim}_{H}\left(\pi_{\Phi}\left(\Lambda_{\mathrm{r}}(N)\right)\right) \geq \operatorname{dim}_{H}\left(\pi_{\Phi}\left(\Lambda_{\mathrm{r}}\left(F_{d}\right)\right)\right) / 2
$$

and strict inequality holds if $(N, \Phi)$ is symmetric.

(4) Suppose that $(N, \Phi)$ is of divergence type.

(a) Then $F_{d} / N$ is amenable.

(b) We have $\operatorname{dim}_{H}\left(\pi_{\Phi}\left(\Lambda_{\mathrm{r}}(N)\right)\right)=\operatorname{dim}_{H}\left(\pi_{\Phi}\left(\Lambda_{\mathrm{r}}\left(F_{d}\right)\right)\right)$ if and only if $(N, \Phi)$ is symmetric on average.

Our next goal is to investigate the set $\pi_{\Phi}\left(\Lambda_{\mathrm{r}}(N) \cap \mathcal{F}(\alpha, \Phi, \psi)\right)$. To this end, let us define

$$
\alpha_{-}:=\inf \{\alpha \in \mathbb{R}: \mathcal{F}(\alpha, \Phi, \psi) \neq \varnothing\} \quad \text { and } \quad \alpha_{+}:=\sup \{\alpha \in \mathbb{R}: \mathcal{F}(\alpha, \Phi, \psi) \neq \varnothing\} .
$$

By Hölder's inequality we see that the free energy function $t_{N}: \mathbb{R} \rightarrow \mathbb{R}$ of $(N, \Phi, \psi)$ is convex. For $\beta \in \mathbb{R}$, we denote by $\partial t_{N}(\beta)$ the subdifferential of $t_{N}$ at $\beta$ ([Roc70, Section 23]), and we set $\partial t_{N}(A):=\bigcup_{\beta \in A} \partial t_{N}(\beta)$, for $A \subset \mathbb{R}$. Let $\operatorname{Int}(A)$ denote the interior of a set $A \subset \mathbb{R}$. We will always assume that $\alpha_{-}<\alpha_{+}$, which is equivalent to the assumption that, for each $\left(a_{1}, a_{2}\right) \in \mathbb{R}^{2} \backslash\{0\}$, the unique Gibbs measures associated with $a_{1} \zeta$ and $a_{2} \psi$ are distinct ([Rue78, PW97]). Then it is well known that $t$ is a real-analytic and strictly convex function which satisfies $-\partial t(\mathbb{R})=\left(\alpha_{-}, \alpha_{+}\right)$. Moreover, we have that $\mathcal{F}(\alpha, \Phi, \psi)=\varnothing$ if and only if $\alpha \notin$ $\left[\alpha_{-}, \alpha_{+}\right]$(Sch99]). Note that, if $\alpha_{-}$and $\alpha_{+}$coincide, then $\mathcal{F}\left(\alpha_{-}, \Phi, \psi\right)=\Sigma$ and the analysis given in Theorem 1.1 applies.

To state our next main result, we define the convex conjugate of $t_{N}$ ( Roc70, Section 12]) given by

$$
t_{N}^{*}: \mathbb{R} \rightarrow \mathbb{R} \cup\{\infty\}, \quad t_{N}^{*}(\alpha):=\sup _{\beta \in \mathbb{R}}\left\{\beta \alpha-t_{N}(\beta)\right\}, \quad \alpha \in \mathbb{R} .
$$


Theorem 1.2. Let $\Phi$ denote a conformal graph directed Markov system associated with $F_{d}, d \geq 2$. Let $\psi: \Sigma \rightarrow \mathbb{R}$ be Hölder continuous. Suppose that $\alpha_{-}<\alpha_{+}$. Then the following holds for each non-trivial normal subgroup $N$ of $F_{d}$.

(1) For each $\alpha \in-\operatorname{Int}\left(\partial t_{N}(\mathbb{R})\right) \subset\left(\alpha_{-}, \alpha_{+}\right)$we have $\operatorname{dim}_{H}\left(\pi_{\Phi}\left(\Lambda_{\mathrm{ur}}(N) \cap \mathcal{F}(\alpha, \Phi, \psi)\right)\right)=\operatorname{dim}_{H}\left(\pi_{\Phi}\left(\Lambda_{\mathrm{r}}(N) \cap \mathcal{F}(\alpha, \Phi, \psi)\right)\right)=-t_{N}^{*}(-\alpha)>0$.

If $(N, \Phi, \psi)$ is asymptotically symmetric, then $-\operatorname{Int}\left(\partial t_{N}(\mathbb{R})\right)=\left(\alpha_{-}, \alpha_{+}\right)$.

(2) If $F_{d} / N$ is non-amenable, then for each $\alpha \in\left(\alpha_{-}, \alpha_{+}\right)$,

$$
\operatorname{dim}_{H}\left(\pi_{\Phi}\left(\Lambda_{\mathrm{r}}(N) \cap \mathcal{F}(\alpha, \Phi, \psi)\right)\right)<\operatorname{dim}_{H}\left(\pi_{\Phi}(\mathcal{F}(\alpha, \Phi, \psi))\right) .
$$

(3) Suppose that $(N, \Phi, \psi)$ is asymptotically symmetric and let $\alpha \in\left(\alpha_{-}, \alpha_{+}\right)$.

(a) If $F_{d} / N$ is amenable, then

$$
\operatorname{dim}_{H}\left(\pi_{\Phi}\left(\Lambda_{\mathrm{r}}(N) \cap \mathcal{F}(\alpha, \Phi, \psi)\right)\right)=\operatorname{dim}_{H}\left(\pi_{\Phi}(\mathcal{F}(\alpha, \Phi, \psi))\right) .
$$

(b) We have

$$
\operatorname{dim}_{H}\left(\pi_{\Phi}\left(\Lambda_{\mathrm{r}}(N) \cap \mathcal{F}(\alpha, \Phi, \psi)\right)\right) \geq \operatorname{dim}_{H}\left(\pi_{\Phi}(\mathcal{F}(\alpha, \Phi, \psi))\right) / 2
$$

and strict inequality holds if $(N, \Phi, \psi)$ is symmetric.

(4) Let $\beta \in \mathbb{R}$ and suppose that $(N, \Phi, \psi)$ is of divergence type in $\beta$.

(a) Then $F_{d} / N$ is amenable.

(b) If $\alpha \in-\partial t(\beta)$ and

$\operatorname{dim}_{H}\left(\pi_{\Phi}\left(\Lambda_{\mathrm{r}}(N) \cap \mathcal{F}(\alpha, \Phi, \psi)\right)\right)=\operatorname{dim}_{H}\left(\pi_{\Phi}(\mathcal{F}(\alpha, \Phi, \psi))\right)$,

then $(N, \Phi, \psi)$ is symmetric on average in $\beta$.

(c) If $\alpha \in-\left(\partial t_{N}(\beta) \cap \operatorname{Int}\left(\partial t_{N}(\mathbb{R})\right)\right)$ and $(N, \Phi, \psi)$ is symmetric on average in $\beta$, then

$$
\operatorname{dim}_{H}\left(\pi_{\Phi}\left(\Lambda_{\mathrm{r}}(N) \cap \mathcal{F}(\alpha, \Phi, \psi)\right)\right)=\operatorname{dim}_{H}\left(\pi_{\Phi}(\mathcal{F}(\alpha, \Phi, \psi))\right) .
$$

Remark 1.3. If $N$ is finitely generated, then $(N, \Phi)$ is of divergence type by Lemma 3.14. Hence, $F_{d} / N$ is amenable by Theorem 1.1 (4a). In fact, it is well known that, if $N$ is a finitely generated normal subgroup of $F_{d}$, then $F_{d} / N$ is finite. In particular, we then have that $\Lambda_{\mathrm{ur}}(N)=\Lambda_{\mathrm{r}}\left(F_{d}\right)=\pi_{\Phi}(\Sigma)$.

Remark 1.4. It is also worth noting that the proof of (11) in Theorems 1.1 and 1.2 makes use of a certain induced graph directed Markov system $\tilde{\Phi}$ (Definition 3.9). This system $\tilde{\Phi}$ is regular ([MU03. Section 4, page 78]) if and only if $(N, \Phi)$ is of divergence type by Lemma 3.14. In particular, we have by Theorem 1.1 (4a) that, if $F_{d} / N$ is non-amenable, then $\tilde{\Phi}$ is irregular.

Before we proceed to discuss applications to Kleinian groups, let us briefly point out a relation to the cogrowth of group presentations $\left\langle g_{1}, \ldots, g_{d} \mid r_{1}, r_{2}, \ldots\right\rangle$ on $d \geq 2$ generators (Gri80, Coh82]). If the geometric potential of $\Phi$ is constant, then it is easy to see that the cogrowth $\eta$ of $\left\langle g_{1}, \ldots, g_{d} \mid r_{1}, r_{2}, \ldots\right\rangle$ is given by $\delta_{N} / \delta$, where $N$ is the normal subgroup generated by $r_{1}, r_{2}, \ldots$. Since every constant potential is symmetric with respect to $N$, the criterion for $\delta_{N}=\delta$ in Theorem 1.1 (2) and (3a) is the well-known cogrowth criterion $\eta=1$ for amenability of $F_{d} / N$, and the lower bound in Theorem $1.2(3 \mathrm{~b})$ corresponds to the lower bound $\eta>1 / 2$ for the cogrowth (Gri80, Coh82]). 
1.1. Related results for Kleinian groups. Let us now state the analogous results for Kleinian groups, which have motivated our results. We first recall some basic notations for Kleinian groups. For references on limit sets of Kleinian groups and the associated hyperbolic manifolds, we refer the reader to Bea95, Mas88, Nic89, MT98, Str06.

For a Kleinian group $\Gamma$ acting on the Poincaré disc model $\mathbb{D}:=\left\{z \in \mathbb{R}^{n+1}:\|z\|<1\right\}$ of hyperbolic $(n+1)$-space, $n \in \mathbb{N}$, the Poincaré series is, for each $s \in \mathbb{R}$, given by $P(\Gamma, s):=\sum_{\gamma \in \Gamma} \mathrm{e}^{-s d(0, \gamma(0))}$, where $d$ denotes the hyperbolic metric on $\mathbb{D}$. The exponent of convergence of $\Gamma$ is given by $\delta(\Gamma):=\inf \{s \geq 0: P(\Gamma, s)<\infty\}$. It is well known by a theorem of Bishop and Jones (BJ97]) that the exponent of convergence of a non-elementary Kleinian group $\Gamma$ is equal to the Hausdorff dimension of the (uniformly) radial limit set of $\Gamma$, that is,

$$
\delta(\Gamma)=\operatorname{dim}_{H}\left(L_{\mathrm{ur}}(\Gamma)\right)=\operatorname{dim}_{H}\left(L_{\mathrm{r}}(\Gamma)\right) .
$$

Passing from a non-elementary Kleinian group $\Gamma$ to a normal subgroup $N$ of $\Gamma$ gives rise to a normal covering of the associated hyperbolic manifolds. Brooks proved in Bro85 that if $\Gamma$ is convex cocompact and $\delta(\Gamma)>n / 2$, then

$$
\delta(\Gamma)=\delta(N) \text { if and only if } \Gamma / N \text { is amenable. }
$$

A recent result of Stadlbauer (Sta13) shows that the amenability dichotomy in (1.2) holds for all essentially free Kleinian groups $\Gamma$ with arbitrary exponent of convergence.

A complementary result is due to Falk and Stratmann ([FS04, Theorem 2]) which states that for each non-trivial normal subgroup $N$ of a non-elementary Kleinian group $\Gamma$, we have that

$$
\delta(N) \geq \delta(\Gamma) / 2 .
$$

Roblin ([Rob05]) proved that strict inequality in (1.3) holds if the Kleinian group $\Gamma$ is of divergence type, that is, $P(\Gamma, \delta(\Gamma))=\infty$. Another proof of this result was independently obtained by Bonfert-Taylor, Matsuzaki and Taylor ([BTMT12]), if $\Gamma$ is convex cocompact. A related result by Matsuzaki and Yabuki in MY09] states that

$$
\text { if } P(N, \delta(N))=\infty \text {, then } \delta(\Gamma)=\delta(N) \text {. }
$$

In Jae13, the author used this result of Matsuzaki and Yabuki to give a short new proof of the strict inequality in (1.3) if $\Gamma$ is of divergence type.

Remark. A related result is due to Rees ([Ree81a, Ree81b]) which shows the following for a non-trivial normal subgroup $N$ of a convex cocompact geometrically finite Fuchsian groups $\Gamma$ such that $\Gamma / N \simeq \mathbb{Z}^{d}$ for some $d \in \mathbb{N}$ : The critical exponents $\delta(N)$ and $\delta(\Gamma)$ coincide. Moreover, we have that $N$ is of divergence type if and only if $d \leq 2$.

We first observe that Theorem 1.1(1) gives the analog statement to (1.1). Moreover, the results given in Theorem 1.1 (2) and (3a) extend the amenability dichotomy in (1.2). The lower bound in Theorem 1.1 (3b) corresponds to (1.3) and strict inequality holds because the finitely generated group $F_{d}$ is of divergence type by Lemma 3.14. Theorem 1.1 (4a) and (4b) shed new light on (1.4). Finally, the results of Theorem 1.2 show that similar results hold if the radial limit set is intersected with 
the level set of a multifractal decomposition with respect to a Hölder continuous potential.

Let us also remark that the symmetry assumptions imposed on the graph directed Markov system $\Phi$ in Theorems 1.1 and 1.2 mimic the property that $d(0, g(0))=$ $d\left(0, g^{-1}(0)\right)$ for every isometry $g$ with respect to the hyperbolic metric $d$. This relation will be further illustrated in the following subsection.

1.1.1. Application to normal subgroups of Kleinian groups of Schottky type. Let us briefly describe a class of Kleinian groups, to which our results are directly applicable. Let $\Gamma=\left\langle\gamma_{1}, \ldots, \gamma_{d}\right\rangle$ denote a Kleinian group of Schottky type with $d \geq 2$. Then $\Gamma$ is a free group generated by hyperbolic transformations $\gamma_{1}, \ldots, \gamma_{d}$ ([Jae14a, Definition 5.2]). Let $N$ denote a non-trivial normal subgroup of $\Gamma$. It is shown in [Jae14a, Proposition 5.6] that there exists a conformal graph directed Markov system $\Phi_{\Gamma}$ associated with $\Gamma=\left\langle\gamma_{1}, \ldots, \gamma_{d}\right\rangle$, such that for each non-trivial normal subgroup $N$ of $\Gamma$,

$$
L_{\mathrm{r}}(N)=\pi_{\Phi_{\Gamma}}\left(\Lambda_{\mathrm{r}}(N)\right) \quad \text { and } \quad L_{\mathrm{ur}}(N)=\pi_{\Phi_{\Gamma}}\left(\Lambda_{\mathrm{ur}}(N)\right) .
$$

The limit set $L(\Gamma)$ is equal to the limit set of $\Phi_{\Gamma}$ and a symbolic representation is given by $\Sigma:=\left\{\tau \in I^{\mathbb{N}}: \tau_{i} \neq \tau_{i+1}^{-1}\right\}$ where $I:=\left\{\gamma_{1}, \gamma_{1}^{-1}, \ldots, \gamma_{d}, \gamma_{d}^{-1}\right\}$. The geometric potential $\zeta: \Sigma \rightarrow \mathbb{R}^{-}$of $\Phi_{\Gamma}$ has the property that there exists $C>0$ such that for all $\omega \in F_{d}$,

$$
C^{-1} \mathrm{e}^{S_{\omega} \zeta} \leq \mathrm{e}^{-d(0, \omega(0))} \leq C \mathrm{e}^{S_{\omega} \zeta} .
$$

We refer to (see Ser81]) for details. Let $t_{N}: \mathbb{R} \rightarrow \mathbb{R}$ denote the free energy function of $\left(N, \Phi_{\Gamma},-1\right)$. Observe that by (1.6) we have for each $\beta \in \mathbb{R}$,

$$
t_{N}(\beta)=\inf \left\{u \in \mathbb{R}: \sum_{\omega \in N} \mathrm{e}^{-\beta|\omega|-u d(0, \omega(0))}<\infty\right\} .
$$

We now consider the (inverse) Lyapunov spectrum of $L(\Gamma)$ and its restriction to $L_{r}(N)$. More precisely, we define for each $\alpha \in \mathbb{R}$ the level sets

$$
\mathcal{L}(\alpha):=\left\{\tau \in L(\Gamma): \lim _{n \rightarrow \infty} \frac{n}{d\left(0, \tau_{1} \ldots \tau_{n}(0)\right)}=\alpha\right\} \quad \text { and } \quad \mathcal{L}_{N}(\alpha):=\mathcal{L}(\alpha) \cap L_{r}(N) .
$$

Set $\alpha_{-}:=\min \{\alpha \in \mathbb{R}: \mathcal{L}(\alpha) \neq \varnothing\}$ and $\alpha_{+}:=\max \{\alpha \in \mathbb{R}: \mathcal{L}(\alpha) \neq \varnothing\}$. As a corollary of Theorem 1.2 , we obtain the following for the Lyapunov spectrum.

Corollary 1.5. Let $\Gamma$ denote a Kleinian group of Schottky type and let $N$ denote a non-trivial normal subgroup of $\Gamma$. Then for each $\alpha \in\left(\alpha_{-}, \alpha_{+}\right)$we have that

$$
\frac{\operatorname{dim}_{H}(\mathcal{L}(\alpha))}{2}<\operatorname{dim}_{H}\left(\mathcal{L}_{N}(\alpha)\right) \leq \operatorname{dim}_{H}(\mathcal{L}(\alpha)) .
$$

The second inequality in (1.7) is an equality if and only if $\Gamma / N$ is amenable. Moreover, if there exists $\beta \in \mathbb{R}$ such that $\sum_{\omega \in N} \mathrm{e}^{-\beta|\omega|-t_{N}(\beta) d(0, \omega(0))}=\infty$, then $\Gamma / N$ is amenable.

Proof. Recall that by (1.5), we have $L_{\mathrm{r}}(N)=\pi_{\Phi_{\Gamma}}\left(\Lambda_{\mathrm{r}}(N)\right)$. Furthermore, we observe that by (1.6) we have $\mathcal{L}(\alpha)=\pi_{\Phi_{\Gamma}}\left(\mathcal{F}\left(\alpha, \Phi_{\Gamma},-1\right)\right)$ where $1: \Sigma \rightarrow\{1\}$. Since $\pi_{\Phi_{\Gamma}}$ is one-to-one we have that $\mathcal{L}_{N}(\alpha)=\pi_{\Phi_{\Gamma}}\left(\mathcal{F}\left(\alpha, \Phi_{\Gamma},-1\right) \cap \Lambda_{\mathrm{r}}(N)\right)$. Using that $d(0, \omega(0))=d\left(0, \omega^{-1}(0)\right)$ for each $\omega \in F_{d}$, we have $C^{-1} \mathrm{e}^{S_{\omega} \zeta} \leq C \mathrm{e}^{S_{\omega-1} \zeta}$ by (1.6). Thus, we have that $\left(N, \Phi_{\Gamma},-1\right)$ is symmetric. The corollary now follows from Theorem 1.2 . 
1.2. Plan of the paper. The results stated in Theorems 1.1 and 1.2 are deduced from general results of the thermodynamic formalism for group-extended Markov systems developed by Stadlbauer ([Sta13] ) and by the author ([Jae11,Jae12, Jae14b, see also [Jae14a]). These results are given in Section 4. The general results stated in Proposition 4.8, which are used to derive (3b) of Theorems 1.1 and 1.2 , are new in the context of group-extended Markov systems. To obtain the strict inequality in (3b) of Theorems 1.1 and 1.2 and the results involving the divergence of the Poincaré series of the normal subgroup, we make use of a characterisation of recurrent potentials from [Jae12].

In Proposition 3.13 we develop a multifractal formalism for the multifractal decomposition of the radial limit set parametrised by a normal subgroup. We make use of an induced graph directed Markov system (Definition 3.9) which is generated by infinitely many maps if the normal subgroup is of infinite index. A similar argument as in KMS12 allows us to relate the limit set of the induced graph directed Markov system to the level sets $\mathcal{F}(\alpha, \Phi, \psi)$ (see Proposition 3.13). We then use the methods from [JK11] to establish the multifractal formalism.

For a graph directed Markov system $\Phi$ consisting of similarities, the results stated in Theorem 1.1 (1), (2), (3a) and (3b) are contained in [Jae14a. In the present paper, we have generalised the results essentially in two ways: First, Theorem 1.1 applies to arbitrary conformal graph directed Markov systems, and second, Theorem 1.2 allows us to investigate intersections of the radial limit set parametrised by a normal subgroup with level sets of a multifractal decomposition with respect to a Hölder continuous potential. Moreover, the result stated in Theorem 1.1 (4b) is new even if $\Phi$ consists of similarities.

The outline of this paper is as follows. In Section 2, we collect the necessary preliminaries on the symbolic thermodynamic formalism for Markov shifts. In Section 3 , we give the definition of conformal graph directed Markov systems associated with free groups and their radial limit sets, and we develop a multifractal formalism in this context. In Section 4 we give results on amenability and recurrence for groupextended Markov systems, from which we deduce our main results in Section 5 .

\section{ThermodynAmiC FORMALISM FOR MARKOV SHIFTS}

Throughout, the state space of the thermodynamic formalism will be a Markov shift $\Sigma$ given by

$$
\Sigma:=\left\{\tau:=\left(\tau_{1}, \tau_{2}, \ldots\right) \in I^{\mathbb{N}}: a\left(\tau_{i}, \tau_{i+1}\right)=1 \text { for all } i \in \mathbb{N}\right\},
$$

where $I$ denotes a finite or countable alphabet, the matrix $A=(a(i, j)) \in\{0,1\}^{I \times I}$ is the incidence matrix and the left shift map $\sigma: \Sigma \rightarrow \Sigma$ is given by $\sigma\left(\tau_{1}, \tau_{2}, \ldots\right):=$ $\left(\tau_{2}, \tau_{3}, \ldots\right)$, for each $\tau \in \Sigma$. We denote by

$$
\Sigma^{n}:=\left\{\omega \in I^{n}: a\left(\omega_{i}, \omega_{i+1}\right)=1,1 \leq i \leq n-1\right\}
$$

the set of $A$-admissible words of length $n \in \mathbb{N}$. The set of $A$-admissible words of arbitrary length is given by $\Sigma^{*}:=\bigcup_{n \in \mathbb{N}} \Sigma^{n}$. We define the word length function $|\cdot|: \Sigma^{*} \cup \Sigma \rightarrow \mathbb{N} \cup\{\infty\}$, where for $\omega \in \Sigma^{*}$ we set $|\omega|$ to be the unique $n \in \mathbb{N}$ such that $\omega \in \Sigma^{n}$ and for $\omega \in \Sigma$ we set $|\omega|:=\infty$. For each $\omega \in \Sigma^{*} \cup \Sigma$ and $n \in \mathbb{N}$ with $1 \leq n \leq|\omega|$, we define $\omega_{\mid n}:=\left(\omega_{1}, \ldots, \omega_{n}\right)$. For $\tau, \tau^{\prime} \in \Sigma$, we let $\tau \wedge \tau^{\prime}:=\tau_{\mid l}$, where 
$l:=\sup \left\{n \in \mathbb{N}: \tau_{\mid n}=\tau_{\mid n}^{\prime}\right\}$. For $n \in \mathbb{N}_{0}$ and $\omega \in \Sigma^{n}$, the cylinder set $[\omega]$ is given by $[\omega]:=\left\{\tau \in \Sigma: \tau_{\mid n}=\omega\right\}$.

If $\Sigma$ is the Markov shift with alphabet $I$ whose incidence matrix consists entirely of $1 \mathrm{~s}$, then we have that $\Sigma=I^{\mathbb{N}}$ and $\Sigma^{n}=I^{n}$, for all $n \in \mathbb{N}$. Then we set $I^{*}:=\Sigma^{*}$. For $\omega, \omega^{\prime} \in I^{*}$ we denote by $\omega \omega^{\prime} \in I^{*}$ the concatenation of $\omega$ and $\omega^{\prime}$, which is defined by $\omega \omega^{\prime}:=\left(\omega_{1}, \ldots, \omega_{|\omega|}, \omega_{1}^{\prime}, \ldots, \omega_{\left|\omega^{\prime}\right|}^{\prime}\right)$ for $\omega, \omega^{\prime} \in I^{*}$. Note that $I^{*}$ forms a semigroup with respect to the concatenation operation. The semigroup $I^{*}$ is the free semigroup generated by $I$ and satisfies the universal property that, for each semigroup $S$ and for every map $u: I \rightarrow S$, there exists a unique semigroup homomorphism $\widehat{u}: I^{*} \rightarrow S$ such that $\widehat{u}(i)=u(i)$, for all $i \in I$.

We equip $I^{\mathbb{N}}$ with the product topology of the discrete topology on $I$. The Markov shift $\Sigma \subset I^{\mathbb{N}}$ is equipped with the subspace topology. A countable basis of this topology on $\Sigma$ is given by the cylinder sets $\left\{[\omega]: \omega \in \Sigma^{*}\right\}$. We will make use of the following metrics generating the product topology on $\Sigma$. For $\alpha>0$ fixed, we define the metric $d_{\alpha}: \Sigma \times \Sigma \rightarrow \mathbb{R}$ on $\Sigma$ given by

$$
d_{\alpha}\left(\tau, \tau^{\prime}\right):=\mathrm{e}^{-\alpha\left|\tau \wedge \tau^{\prime}\right|}, \text { for all } \tau, \tau^{\prime} \in \Sigma .
$$

For a function $\varphi: \Sigma \rightarrow \mathbb{R}$ and $n \in \mathbb{N}_{0}$ we use the notation $S_{n} \varphi: \Sigma \rightarrow \mathbb{R}$ to denote the ergodic sum of $\varphi$ with respect to $\sigma$, in other words, $S_{n} \varphi:=\sum_{i=0}^{n-1} \varphi \circ \sigma^{i}$.

We say that a function $\varphi: \Sigma \rightarrow \mathbb{R}$ is $\alpha$-Hölder continuous, for some $\alpha>0$, if

$$
V_{\alpha}(\varphi):=\sup _{n \geq 1}\left\{V_{\alpha, n}(\varphi)\right\}<\infty,
$$

where for each $n \in \mathbb{N}$ we let

$$
V_{\alpha, n}(\varphi):=\sup \left\{\frac{\left|\varphi(\tau)-\varphi\left(\tau^{\prime}\right)\right|}{d_{\alpha}\left(\tau, \tau^{\prime}\right)}: \tau, \tau^{\prime} \in \Sigma,\left|\tau \wedge \tau^{\prime}\right| \geq n\right\}
$$

The function $\varphi$ is Hölder continuous if there exists $\alpha>0$ such that $\varphi$ is $\alpha$-Hölder continuous.

The following fact is well known.

Fact 2.1 (MU03, Lemma 2.3.1]). If $\varphi: \Sigma \rightarrow \mathbb{R}$ is Hölder continuous, then there exists a constant $C_{\varphi}>0$ such that, for all $\omega \in \Sigma^{*}$ and $\tau, \tau^{\prime} \in[\omega]$, we have

$$
\left|S_{|\omega|} \varphi(\tau)-S_{|\omega|} \varphi\left(\tau^{\prime}\right)\right| \leq C_{\varphi}
$$

We will make use of the following notion of pressure introduced in [JKL14, Definition 1.1].

Definition 2.2 (Induced topological pressure). For $\varphi, \Delta: \Sigma \rightarrow \mathbb{R}$ with $\Delta \geq 0$, and $\mathcal{C} \subset \Sigma^{*}$ we define for $\eta>0$ the $\Delta$-induced pressure of $\varphi$ (with respect to $\mathcal{C}$ ) by

$$
\mathcal{P}_{\Delta}(\varphi, \mathcal{C}):=\limsup _{T \rightarrow \infty} \frac{1}{T} \log \sum_{\substack{\omega \in \mathcal{C} \\ T-\eta<S_{\omega} \Delta \leq T}} \mathrm{e}^{S_{\omega} \varphi},
$$

which takes values in $\mathbb{R} \cup\{ \pm \infty\}$. In here, we set $S_{\omega} \varphi:=\sup _{\tau \in[\omega]} S_{|\omega|} \varphi(\tau)$.

Remark. It was shown in [JKL14, Theorem 2.4] that the definition of $\mathcal{P}_{\Delta}(\varphi, \mathcal{C})$ is in fact independent of the choice of $\eta>0$. For this reason we do not refer to $\eta>0$ in the definition of the induced pressure. 
Notation. If $\Delta$ and/or $\mathcal{C}$ is left out in the notation of induced pressure, then we tacitly assume that $\Delta=1$ and/or $\mathcal{C}=\Sigma^{*}$. That is, $\mathcal{P}(\varphi):=\mathcal{P}_{1}\left(\varphi, \Sigma^{*}\right)$.

We will make use of the following mixing properties for a Markov shift $\Sigma$ with alphabet $I$.

- $\Sigma$ is irreducible if, for all $i, j \in I$, there exists $\omega \in \Sigma^{*}$ such that $i \omega j \in \Sigma^{*}$.

- $\Sigma$ is topologically mixing if, for all $i, j \in I$, there exists $n_{0} \in \mathbb{N}$ with the property that, for all $n \geq n_{0}$, there exists $\omega \in \Sigma^{n}$ such that $i \omega j \in \Sigma^{*}$.

- $\Sigma$ is finitely irreducible if there exists a finite set $F \subset \Sigma^{*}$ with the property that, for all $i, j \in I$, there exists $\omega \in F$ such that $i \omega j \in \Sigma^{*}$.

- $\Sigma$ is finitely primitive if there exists $l \in \mathbb{N}$ and a finite set $F \subset \Sigma^{l}$ with the property that, for all $i, j \in I$, there exists $\omega \in F$ such that $i \omega j \in \Sigma^{*}$.

Remark. Note that $\Sigma$ is finitely primitive if and only if $\Sigma$ is topologically mixing and if $\Sigma$ satisfies the big images and preimages property (see [Sar03]).

The following fact is taken from [JKL14, Section 2]. (The characterisation of induced pressure via the exponent of convergence of the Poincaré series is proved in [JKL14, Theorem 2.4 and Remark 2.7], for the other properties, see [JKL14, Corollary 2.10 and Remark 2.11].)

Fact 2.3. Let $\Sigma$ be finitely irreducible, $\mathcal{C} \subset \Sigma^{*}$ and let $\varphi, \Delta: \Sigma \rightarrow \mathbb{R}$ with $\Delta \geq c>0$ for some $c>0$. Then we have

$$
\mathcal{P}_{\Delta}(\varphi, \mathcal{C})=\inf \{u \in \mathbb{R}: \mathcal{P}(\varphi-u \Delta, \mathcal{C}) \leq 0\}=\inf \left\{u \in \mathbb{R}: \sum_{\omega \in \mathcal{C}} \mathrm{e}^{S_{\omega}(\varphi-u \Delta)}<\infty\right\} .
$$

If additionally card $(I)<\infty$, then $u \mapsto \mathcal{P}(\varphi-u \Delta, \mathcal{C})$ defines a strictly decreasing, continuous map on $\mathbb{R}$ with values in $\mathbb{R}$. Moreover, we have that $\mathcal{P}_{\Delta}(\varphi, \mathcal{C})$ is the unique $u \in \mathbb{R}$ such that $\mathcal{P}(\varphi-u \Delta, \mathcal{C})=0$.

The following notion of a Gibbs measure is fundamental for the thermodynamic formalism (cf. Rue69, Bow75]).

Definition 2.4 (Gibbs measure). Let $\varphi: \Sigma \rightarrow \mathbb{R}$ be continuous. We say that a Borel probability measure $\mu$ is a Gibbs measure for $\varphi$ if there exists a constant $C_{\mu}>0$ such that

$$
C_{\mu}^{-1} \leq \frac{\mu([\omega])}{\mathrm{e}^{S_{|\omega|} \varphi(\tau)-|\omega| \mathcal{P}(\varphi)}} \leq C_{\mu}, \text { for all } \omega \in \Sigma^{*} \text { and } \tau \in[\omega]
$$

The following theorem is proved in [MU03, Section 2].

Theorem 2.5 (Existence of Gibbs measures). Let $\Sigma$ be finitely irreducible and let $\varphi: \Sigma \rightarrow \mathbb{R}$ be Hölder continuous with $\mathcal{P}(\varphi)<\infty$. Then there exists a unique $\sigma$-invariant Gibbs measure for $\varphi$.

\section{Graph directed Markov systems associated With free Groups}

In this section we first recall the definition of a conformal graph directed Markov system (GDMS) introduced by Mauldin and Urbański (MU03]). Then we give the definition of a GDMS associated with a free group and the radial limit set ([Jae14a $)$. 


\subsection{Preliminaries.}

Definition 3.1 (Graph directed Markov system, [MU03). A graph directed Markov system $(G D M S) \Phi=\left(V,\left(X_{v}\right)_{v \in V}, E, i, t,\left(\phi_{e}\right)_{e \in E}, A\right)$ consists of a finite vertex set $V$, a family of non-empty compact metric spaces $\left(X_{v}\right)_{v \in V}$, a countable edge set $E$, maps $i, t: E \rightarrow V$, a family of injective contractions $\phi_{e}: X_{t(e)} \rightarrow X_{i(e)}$ with Lipschitz constants bounded by some $0<s<1$, and an edge incidence matrix $A \in\{0,1\}^{E \times E}$, which has the property that $a(e, f)=1$ implies $t(e)=i(f)$, for all $e, f \in E$. The coding map of $\Phi$ is given by

$$
\pi_{\Phi}: \Sigma_{\Phi} \rightarrow \bigoplus_{v \in V} X_{v}, \text { such that } \bigcap_{n \in \mathbb{N}} \phi_{\tau_{\mid n}}\left(X_{t\left(\tau_{n}\right)}\right)=\left\{\pi_{\Phi}(\tau)\right\} \text {, for each } \tau \in \Sigma_{\Phi},
$$

where $\bigoplus_{v \in V} X_{v}$ denotes the disjoint union of the sets $X_{v}$ and $\Sigma_{\Phi}$ denotes the Markov shift with alphabet set $E$ and incidence matrix $A$. The limit set of $\Phi$ is defined by $J(\Phi):=\pi_{\Phi}\left(\Sigma_{\Phi}\right)$. Further, we set

$$
J^{*}(\Phi):=\bigcup_{F \subset E, \operatorname{card}(F)<\infty} \pi_{\Phi}\left(\Sigma_{\Phi} \cap F^{\mathbb{N}}\right) .
$$

Definition 3.2 (Conformal GDMS, MU03). The GDMS $\Phi=\left(V,\left(X_{v}\right)_{v \in V}, E, i, t\right.$, $\left.\left(\phi_{e}\right)_{e \in E}, A\right)$ is called conformal if the following conditions are satisfied.

(a) For $v \in V$, the phase space $X_{v}$ is a compact connected subset of the Euclidean space $\left(\mathbb{R}^{D},\|\cdot\|\right)$, for some $D \geq 1$, such that $X_{v}$ is equal to the closure of its interior, that is, $X_{v}=\overline{\operatorname{Int}\left(X_{v}\right)}$.

(b) (Open set condition (OSC)). For all $a, b \in E$ with $a \neq b$, we have that

$$
\phi_{a}\left(\operatorname{Int}\left(X_{t(a)}\right)\right) \cap \phi_{b}\left(\operatorname{Int}\left(X_{t(b)}\right)\right)=\varnothing .
$$

(c) For each vertex $v \in V$ there exists an open connected set $W_{v} \supset X_{v}$ such that the map $\phi_{e}$ extends to a $C^{1}$ conformal diffeomorphism of $W_{v}$ into $W_{i(e)}$, for every $e \in E$ with $t(e)=v$.

(d) (Cone property). There exist $l>0$ and $0<\gamma<\pi / 2$ such that, for each $v \in V$ and $x \in X_{v} \subset \mathbb{R}^{D}$ there exists an open cone $\operatorname{Con}(x, \gamma, l) \subset \operatorname{Int}\left(X_{v}\right)$ with vertex $x$, central angle of measure $\gamma$ and altitude $l$.

(e) There are two constants $L \geq 1$ and $\alpha>0$ such that for each $e \in E$ and $x, y \in X_{t(e)}$ we have

$$
\left\|\phi_{e}^{\prime}(y)|-| \phi_{e}^{\prime}(x)\right\| \leq L \inf _{u \in W_{t(e)}}\left|\phi_{e}^{\prime}(u)\right|\|y-x\|^{\alpha},
$$

where $|\cdot|$ refers to the operator norm of a bounded linear operator on $\left(\mathbb{R}^{D},\|\cdot\|\right)$.

Lemma 3.3 (MU03, Lemma 4.2.2]). If $\Phi$ is a conformal GDMS, then for all $\omega \in \Sigma_{\Phi}^{*}$ and for all $x, y \in W_{t(\omega)}$, we have

$$
|\log | \phi_{\omega}^{\prime}(x)|-\log | \phi_{\omega}^{\prime}(y)\left\|\leq \frac{L}{1-s}\right\| x-y \|^{\alpha} .
$$

Definition 3.4. For a GDMS $\Phi$ satisfying (国) and (ㄷ) of Definition 3.2 the geometric potential $\zeta: \Sigma_{\Phi} \rightarrow \mathbb{R}^{-}$of $\Phi$ is given by

$$
\zeta(\tau):=\log \left|\phi_{\tau_{1}}^{\prime}\left(\pi_{\Phi}(\sigma(\tau))\right)\right|, \text { for all } \tau \in \Sigma_{\Phi} .
$$

The following fact follows from [MU03, Proposition 4.2.7, Lemma 3.1.3] and Lemma 3.3 . 
Fact 3.5. Suppose that a GDMS $\Phi$ satisfies (国) and (ㄷ) of Definition 3.2 and that the inequality (3.1) in Lemma 3.3 holds. Then the geometric potential $\zeta$ of $\Phi$ is Hölder continuous. In particular, $\zeta$ is Hölder continuous if $\Phi$ is a conformal GDMS.

The following result is taken from [MU03, Theorem 4.2.13], where finitely primitivity can be replaced by finitely irreducibility (see also [RU08, Theorem 3.7]). The last equality in Theorem 3.6 follows from Fact 2.3 with $\varphi:=0$ and $\Delta:=-\zeta$, since the geometric potential $\zeta$ of $\Phi$ satisfies $\zeta \leq \log (s)<0$, where $s$ denotes the uniform bound for the Lipschitz constants of the generators of $\Phi$ (see Definition 3.1).

Theorem 3.6 (Generalised Bowen's formula). Let $\Phi$ be a conformal GDMS with a finitely irreducible incidence matrix $A$ and geometric potential $\zeta: \Sigma_{\Phi} \rightarrow \mathbb{R}^{-}$. We then have that

$\operatorname{dim}_{H}(J(\Phi))=\operatorname{dim}_{H}\left(J^{*}(\Phi)\right)=\inf \{u \in \mathbb{R}: \mathcal{P}(u \zeta) \leq 0\}=\inf \left\{u \in \mathbb{R}: \sum_{\omega \in \Sigma_{\Phi}^{*}} \mathrm{e}^{u S_{\omega} \zeta}<\infty\right\}$.

Remark 3.7. The generalised Bowen's formula also holds if the GDMS $\Phi$ satisfies

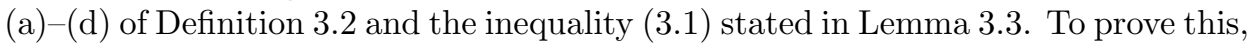
we distinguish two cases. In the case $D \geq 2$, it follows from MU03. Proposition 4.2.1] that, if $\Phi$ satisfies (国) and (IC) of Definition 3.2, then $\Phi$ automatically satisfies (还) with $\alpha=1$. If $D=1$, then a closer inspection of the proof of [MU03, Theorem 4.2.13] shows that Definition 3.2 (还) is in fact only used to deduce (3.1) of Lemma 3.3 (cf. [MU96, Lemma 2.2]).

3.2. Radial limit sets. Graph directed Markov systems associated with free groups and their radial limit sets have been introduced in [Jae14a, Definition 2.10].

Definition 3.8 (GDMS associated with a free group, (uniformly) radial limit set). We denote by $F_{d}=\left\langle g_{1}, \ldots, g_{d}\right\rangle$ the free group on $d \geq 2$ generators. Let

$$
I:=\left\{g_{1}, g_{1}^{-1}, \ldots, g_{d}, g_{d}^{-1}\right\} \quad \text { and } \quad \Sigma:=\left\{\tau \in I^{\mathbb{N}}: \tau_{i} \neq \tau_{i+1}^{-1}\right\} .
$$

Let $N$ be a non-trivial normal subgroup of $F_{d}$. The symbolic radial limit set of $N$ and the symbolic uniformly radial limit set of $N$ (with respect to $F_{d}=\left\langle g_{1}, \ldots, g_{d}\right\rangle$ ) are given by

$$
\Lambda_{\mathrm{r}}(N):=\left\{\tau \in \Sigma: \exists h \in F_{d}, \text { such that } \tau_{1} \cdots \tau_{n} \in h N \text { for infinitely many } n \in \mathbb{N}\right\}
$$

and

$\Lambda_{\text {ur }}(N):=\left\{\tau \in \Sigma: \exists H \subset F_{d}\right.$ finite, such that $\tau_{1} \cdots \tau_{n} \in H N$ for all $\left.n \in \mathbb{N}\right\}$.

A GDMS $\Phi=\left(V,\left(X_{v}\right)_{v \in V}, E, i, t,\left(\phi_{e}\right)_{e \in E}, A\right)$ is associated with $F_{d}=\left\langle g_{1}, \ldots, g_{d}\right\rangle$, $d \geq 2$, if $V=\left\{g_{1}, g_{1}^{-1}, \ldots, g_{d}, g_{d}^{-1}\right\}, E=\left\{(v, w) \in V^{2}: v \neq w^{-1}\right\}, i, t: E \rightarrow V$ are given by $i(v, w)=v$ and $t(v, w)=w$ and the incidence matrix $A \in\{0,1\}^{E \times E}$ satisfies $a(e, f)=1$ if and only if $t(e)=i(f)$, for all $e, f \in E$. We will tacitly apply the canonical bijection between the spaces

$$
\Sigma_{\Phi}=\left\{\left(\left(v_{1}, v_{2}\right),\left(v_{2}, v_{3}\right), \ldots\right) \in(V \times V)^{\mathbb{N}}: v_{i} \neq v_{i+1}^{-1}\right\}
$$

and

$$
\Sigma=\left\{\left(v_{1}, v_{2}, \ldots\right) \in V^{\mathbb{N}}: v_{i} \neq v_{i+1}^{-1}\right\} .
$$

We call $\pi_{\Phi}\left(\Lambda_{\mathrm{r}}(N)\right)$ and $\pi_{\Phi}\left(\Lambda_{\mathrm{ur}}(N)\right)$ the radial limit set of $N$ with respect to $\Phi$ and the uniformly radial limit set of $N$ with respect to $\Phi$. 
3.3. The induced GDMS. In order to investigate the radial limit set of a normal subgroup $N$ of $F_{d}$ with respect to a GDMS $\Phi$ associated with $F_{d}$, we introduce an induced GDMS $\tilde{\Phi}$ whose edge set consists of first return loops in the Cayley graph of $F_{d} / N$.

Definition 3.9. Let $\Phi=\left(V,\left(X_{v}\right)_{v \in V}, E, i, t,\left(\phi_{e}\right)_{e \in E}, A\right)$ denote a conformal GDMS associated with $F_{d}, d \geq 2$, and let $N$ denote a non-trivial normal subgroup of $F_{d}$. The $N$-induced GDMS of $\Phi$ is given by

$$
\tilde{\Phi}:=\left(V,\left(X_{v}\right)_{v \in V}, \tilde{E}, \tilde{i}, \tilde{t},\left(\tilde{\phi}_{\omega}\right)_{\omega \in \tilde{E}}, \tilde{A}\right),
$$

where the edge set $\tilde{E}$ is given by

$\tilde{E}:=\left\{\omega \in \Sigma_{\Phi}^{*}: i\left(\omega_{1}\right) \cdots i\left(\omega_{|\omega|}\right) \in N\right.$ and $i\left(\omega_{1}\right) \cdots i\left(\omega_{k}\right) \notin N$ for $\left.1 \leq k<|\omega|\right\}$, and the maps $\tilde{i}, \tilde{t}: \tilde{E} \rightarrow V$ are given by $\tilde{i}(\omega):=i\left(\omega_{1}\right)$ and $\tilde{t}(\omega):=t\left(\omega_{|\omega|}\right)$, for each $\omega \in \tilde{E}$. Further, the incidence matrix $\tilde{A} \in\{0,1\}^{\tilde{E} \times \tilde{E}}$ is given by $\tilde{a}\left(\omega, \omega^{\prime}\right):=$ $a\left(\omega_{|\omega|}, \omega_{1}^{\prime}\right)$ and the contractions $\left(\tilde{\phi}_{\omega}\right)_{\omega \in \tilde{E}}$ are defined by $\tilde{\phi}_{\omega}:=\phi_{\omega_{1}} \circ \cdots \circ \phi_{\omega_{|\omega|}}$, for each $\omega \in \tilde{E}$.

Notation 3.10. For the $N$-induced GDMS $\tilde{\Phi}$, there are canonical embeddings $\Sigma_{\tilde{\Phi}}^{*} \hookrightarrow$ $\Sigma_{\Phi}^{*}$ and $\Sigma_{\tilde{\Phi}} \hookrightarrow \Sigma_{\Phi}$, which we will both denote by $\iota$. It will always be clear which map is in use.

Definition 3.11. For a function $f: \Sigma_{\Phi} \rightarrow \mathbb{R}$, the induced version $\tilde{f}: \Sigma_{\tilde{\Phi}} \rightarrow \mathbb{R}$ is given by $\tilde{f}(\tilde{\tau}):=S_{\left|\iota\left(\tilde{\tau}_{1}\right)\right|} f(\iota(\tau))$, for each $\tilde{\tau}=\left(\tilde{\tau}_{1}, \tilde{\tau}_{2}, \ldots\right) \in \Sigma_{\tilde{\Phi}}$.

The proof of the following lemma is straightforward and therefore omitted.

Lemma 3.12. Let $\Phi$ denote a conformal GDMS associated with $F_{d}, d \geq 2$. Let $N$ denote a non-trivial normal subgroup of $F_{d}$, and let $\tilde{\Phi}$ denote the $N$-induced GDMS of $\Phi$. Then we have the following.

(1) The incidence matrix $\tilde{A}$ of $\tilde{\Phi}$ is finitely irreducible.

(2) For the coding maps $\pi_{\tilde{\Phi}}: \Sigma_{\tilde{\Phi}} \rightarrow J(\tilde{\Phi})$ and $\pi_{\Phi}: \Sigma_{\Phi} \rightarrow J(\Phi)$, we have $\pi_{\tilde{\Phi}}(\tilde{\tau})=\pi_{\Phi}(\iota(\tilde{\tau}))$ for each $\tilde{\tau} \in \Sigma_{\tilde{\Phi}}$.

(3) The geometric potential $\tilde{\zeta}: \Sigma_{\tilde{\Phi}} \rightarrow \mathbb{R}$ of $\tilde{\Phi}$ is the induced version of the geometric potential $\zeta: \Sigma_{\Phi} \rightarrow \mathbb{R}$ of $\Phi$.

(4) Let $f: \Sigma_{\Phi} \rightarrow \mathbb{R}$ be Hölder continuous. Then the induced version $\tilde{f}$ : $\Sigma_{\tilde{\Phi}} \rightarrow \mathbb{R}$ is Hölder continuous and there exists a constant $C_{f}>0$ such that $S_{\iota(\tilde{\omega})} f-C_{f} \leq S_{\tilde{\omega}} \tilde{f} \leq S_{\iota(\tilde{\omega})} f$, for all $\tilde{\omega} \in \Sigma_{\tilde{\Phi}}^{*}$.

The next proposition provides a version of Bowen's formula for the Hausdorff dimension of the radial limit set of a normal subgroup $N$ of $F_{d}$ with respect to a conformal GDMS $\Phi$ associated with $F_{d}$. This extends [Jae11, Proposition 6.2.8] and [Jae14a, Proposition 1.3]. Moreover, we establish a multifractal formalism in this context.

Proposition 3.13. Let $\Phi$ denote a conformal GDMS associated with $F_{d}, d \geq 2$, and let $N$ denote a non-trivial normal subgroup of $F_{d}$. Let $\psi: \Sigma_{\Phi} \rightarrow \mathbb{R}$ be Hölder continuous and let $t_{N}$ denote the free energy function of $(N, \Phi, \psi)$. Then we have the following. 
(1) $\operatorname{dim}_{H}\left(\pi_{\Phi}\left(\Lambda_{\text {ur }}(N)\right)\right)=\operatorname{dim}_{H}\left(\pi_{\Phi}\left(\Lambda_{\mathrm{r}}(N)\right)\right)=\delta_{N}$.

(2) $\operatorname{dim}_{H}\left(\pi_{\Phi}\left(\Lambda_{\mathrm{r}}(N) \cap \mathcal{F}(\alpha, \Phi, \psi)\right)\right) \leq \max \left\{-t_{N}^{*}(-\alpha), 0\right\}$, for each $\alpha \in \mathbb{R}$.

(3) $\operatorname{dim}_{H}\left(\pi_{\Phi}\left(\Lambda_{\text {ur }}(N) \cap \mathcal{F}(\alpha, \Phi, \psi)\right)\right)=\operatorname{dim}_{H}\left(\pi_{\Phi}\left(\Lambda_{\mathrm{r}}(N) \cap \mathcal{F}(\alpha, \Phi, \psi)\right)\right)=$ $-t_{N}^{*}(-\alpha)>0$, for each $\alpha \in-\operatorname{Int}\left(\partial t_{N}(\mathbb{R})\right)$.

Proof. Let $\tilde{\Phi}$ denote the $N$-induced GDMS of $\Phi$. First, we relate the limit set of $\tilde{\Phi}$ to the radial limit set of $N$ with respect to $\Phi$. Using Lemma 3.12 (2), it is straightforward to verify that

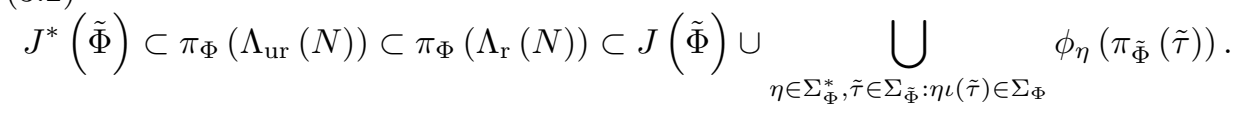

Note that the right-hand side of (3.2) is a countable union of Lipschitz continuous images of $J(\tilde{\Phi})$. Since Lipschitz continuous maps do not increase Hausdorff dimension and since Hausdorff dimension is stable under countable unions, we obtain that

$$
\operatorname{dim}_{H}\left(J^{*}(\tilde{\Phi})\right) \leq \operatorname{dim}_{H}\left(\pi_{\Phi}\left(\Lambda_{\text {ur }}(N)\right)\right) \leq \operatorname{dim}_{H}\left(\pi_{\Phi}\left(\Lambda_{\mathrm{r}}(N)\right)\right) \leq \operatorname{dim}_{H}(J(\tilde{\Phi})) .
$$

The GDMS $\tilde{\Phi}$ satisfies the conditions (国)-(d) in Definition 3.2, Further, since $\left\{\tilde{\phi}_{\tilde{\omega}}: \tilde{\omega} \in \Sigma_{\tilde{\Phi}}^{*}\right\}$ is a subfamily of $\left\{\phi_{\omega}: \omega \in \Sigma_{\Phi}^{*}\right\}$, it follows that $\tilde{\Phi}$ satisfies (3.1) of Lemma 3.3. Moreover, by Lemma 3.12 (11), the incidence matrix of $\tilde{\Phi}$ is finitely irreducible. Hence, by Remark 3.7, the generalised Bowen's formula in Theorem 3.6 and (3.3) give that

$$
\operatorname{dim}_{H}\left(\pi_{\Phi}\left(\Lambda_{\text {ur }}(N)\right)\right)=\operatorname{dim}_{H}\left(\pi_{\Phi}\left(\Lambda_{\mathrm{r}}(N)\right)\right)=\inf \left\{\beta \in \mathbb{R}: \sum_{\tilde{\omega} \in \Sigma_{\tilde{\Phi}}^{*}} \mathrm{e}^{\beta S_{\tilde{\omega}} \tilde{\zeta}}<\infty\right\} .
$$

For each $\tilde{\omega} \in \Sigma_{\tilde{\Phi}}^{*}$ there is $n \in \mathbb{N}$ such that $\iota(\tilde{\omega}) \in \Sigma_{\Phi}^{n}$. Write $\iota(\tilde{\omega})=\left(\left(v_{1}, w_{1}\right),\left(v_{2}, w_{2}\right)\right.$, $\left.\ldots,\left(v_{n}, w_{n}\right)\right)$. By mapping each element $\left(\left(v_{1}, w_{1}\right),\left(v_{2}, w_{2}\right), \ldots,\left(v_{n}, w_{n}\right)\right)$ to $\left(v_{1} v_{2} \ldots v_{n}\right)$, we obtain a $(2 d-1)$-to-one map from $\Sigma_{\tilde{\Phi}}^{*}$ onto $N \backslash\{$ id $\}$. Hence, by Lemma 3.12 (4), we see that

$$
\inf \left\{\beta \in \mathbb{R}: \sum_{\tilde{\omega} \in \Sigma_{\tilde{\Phi}}^{*}} \mathrm{e}^{\beta S \tilde{\omega} \tilde{\zeta}}<\infty\right\}=\inf \left\{\beta \in \mathbb{R}: \sum_{\omega \in N \backslash\{\operatorname{id}\}} \mathrm{e}^{\beta S_{\omega} \zeta}<\infty\right\}=\delta_{N} .
$$

Combining (3.4) and (3.5) finishes the proof of (11).

For the remaining part of the proof, we define for each $\alpha \in \mathbb{R}$ the symbolic level sets

$$
\tilde{\mathcal{F}}^{*}(\alpha):=\left\{\tilde{\tau}=\left(\tilde{\tau}_{1}, \tilde{\tau}_{2}, \ldots\right) \in \Sigma_{\tilde{\Phi}}: \lim _{k \rightarrow \infty} \frac{S_{\left(\tilde{\tau}_{1}, \ldots, \tilde{\tau}_{k}\right)} \tilde{\psi}}{S_{\left(\tilde{\tau}_{1}, \ldots, \tilde{\tau}_{k}\right)} \tilde{\zeta}}=\alpha \text { and } \sup _{i \in \mathbb{N}}\left\{\left|\iota\left(\tilde{\tau}_{i}\right)\right|\right\}<\infty\right\}
$$

and

$$
\tilde{\mathcal{F}}(\alpha):=\left\{\tilde{\tau}=\left(\tilde{\tau}_{1}, \tilde{\tau}_{2}, \ldots\right) \in \Sigma_{\tilde{\Phi}}: \lim _{k \rightarrow \infty} \frac{S_{\left(\tilde{\tau}_{1}, \ldots, \tilde{\tau}_{k}\right)} \tilde{\psi}}{S_{\left(\tilde{\tau}_{1}, \ldots, \tilde{\tau}_{k}\right)} \tilde{\zeta}}=\alpha\right\}
$$


Furthermore, we define $\tilde{t}: \mathbb{R} \rightarrow \mathbb{R} \cup\{\infty\}$ which is for each $\beta \in \mathbb{R}$ given by

$$
\tilde{t}(\beta):=\inf \{u \in \mathbb{R}: \mathcal{P}(\beta \tilde{\psi}+u \tilde{\zeta}) \leq 0\}
$$

By Fact 2.3 we have that for each $\beta \in \mathbb{R}$,

$$
\tilde{t}(\beta)=\inf \left\{u \in \mathbb{R}: \sum_{\tilde{\omega} \in \Sigma_{\tilde{\Phi}}^{*}} \mathrm{e}^{\beta S_{\tilde{\omega}} \tilde{\psi}+u S_{\tilde{\omega}} \tilde{\zeta}}<\infty\right\} .
$$

Following the proof of (3.5), we see that, for each $\beta \in \mathbb{R}$, we have

$$
\tilde{t}(\beta)=t_{N}(\beta) \text {. }
$$

To prove the upper bound for the Hausdorff dimension in (2), first observe that, similarly as in (3.2), the set $\pi_{\Phi}\left(\Lambda_{\mathrm{r}}(N) \cap \mathcal{F}(\alpha, \Phi, \psi)\right)$ is contained in a countable union of Lipschitz continuous images of $\pi_{\tilde{\Phi}}(\tilde{\mathcal{F}}(\alpha))$. Then by a standard covering argument (see e.g. JK11, Theorem 1.2]) we have $\operatorname{dim}_{H}(\tilde{\mathcal{F}}(\alpha)) \leq \max \left\{-\tilde{t}^{*}(-\alpha), 0\right\}$, which, in light of (3.6), finishes the proof of (2).

To prove (3), we first verify that for each $\alpha \in \mathbb{R}$,

$$
\iota\left(\tilde{\mathcal{F}}^{*}(\alpha)\right) \subset \Lambda_{\text {ur }}(N) \cap \mathcal{F}(\alpha, \Phi, \psi) .
$$

Clearly, we have that $\iota\left(\tilde{\mathcal{F}}^{*}(\alpha)\right) \subset \Lambda_{\text {ur }}(N)$, so it remains to show that $\iota\left(\tilde{\mathcal{F}}^{*}(\alpha)\right) \subset$ $\mathcal{F}(\alpha, \Phi, \psi)$. The proof follows [KMS12, Proposition 4.3]. Let $\tilde{\tau} \in \tilde{\mathcal{F}}^{*}(\alpha)$ be given and set $\tau:=\iota(\tilde{\tau})$. Then there exists $l \in \mathbb{N}$ such that $\left|\iota\left(\tilde{\tau}_{i}\right)\right| \leq l$, for each $i \in \mathbb{N}$. For each $n>l$, let

$$
k(n):=\max \left\{k \in \mathbb{N}: \sum_{i=1}^{k} \iota\left(\left|\tilde{\tau}_{i}\right|\right) \leq n\right\} .
$$

Then there exists $r(n) \leq l$ such that $n=\sum_{i=1}^{k(n)}\left|\iota\left(\tilde{\tau}_{i}\right)\right|+r(n)$. For $f \in\{\zeta, \psi\}$ we set

$M_{f}:=\max \left\{S_{\omega} f: \omega \in \Sigma^{r}, 1 \leq r \leq l\right\} \quad$ and $\quad m_{f}:=\min \left\{S_{\omega} f: \omega \in \Sigma^{r}, 1 \leq r \leq l\right\}$. Since $\zeta$ and $\psi$ are Hölder continuous, we have by Lemma 3.12 (4) and Fact 2.1 that

$$
S_{\left(\tilde{\tau}_{1}, \ldots, \tilde{\tau}_{k(n)}\right)} \tilde{f}+m_{f}-2 C_{f} \leq S_{\left(\tau_{1}, \ldots, \tau_{n}\right)} f \leq S_{\left(\tilde{\tau}_{1}, \ldots, \tilde{\tau}_{k(n)}\right)} \tilde{f}+M_{f}+C_{f},
$$

which then gives

$$
\frac{S_{\left(\tilde{\tau}_{1} \ldots . \tilde{\tau}_{k(n)}\right)} \tilde{\psi}+m_{\psi}-2 C_{\psi}}{S_{\left(\tilde{\tau}_{1} \ldots . \tilde{\tau}_{k(n)}\right)} \tilde{\zeta}+M_{\zeta}+C_{\zeta}} \leq \frac{S_{\left(\tau_{1}, \ldots, \tau_{n}\right)} \psi}{S_{\left(\tau_{1}, \ldots, \tau_{n}\right)} \zeta} \leq \frac{S_{\left(\tilde{\tau}_{1} \ldots \ldots \tilde{\tau}_{k(n)}\right)} \tilde{\psi}+M_{\psi}+C_{\psi}}{S_{\left(\tilde{\tau}_{1} \cdots \cdot \tilde{\tau}_{k(n)}\right)} \tilde{\zeta}+m_{\zeta}-2 C_{\zeta}} .
$$

Since $\tilde{\tau} \in \tilde{\mathcal{F}}^{*}(\alpha)$ and $\left|S_{\left(\tilde{\tau}_{1} \cdots \ldots \tilde{\tau}_{k(n)}\right)} \tilde{\zeta}\right| \rightarrow \infty$, as $n \rightarrow \infty$, it follows that $\tau \in$ $\mathcal{F}(\alpha, \Phi, \psi)$. The proof of (3.7) is complete. By Lemma 3.12 (2) we conclude that $\pi_{\tilde{\Phi}}\left(\tilde{\mathcal{F}}^{*}(\alpha)\right) \subset \pi_{\Phi}\left(\Lambda_{\text {ur }}(N) \cap \mathcal{F}(\alpha, \Phi, \psi)\right)$. Combining with the upper bound in (2) and (3.6), the proof will be completed, if we have shown that

$$
\operatorname{dim}_{H}\left(\pi_{\tilde{\Phi}}\left(\tilde{\mathcal{F}}^{*}(\alpha)\right)\right)=-\tilde{t}^{*}(-\alpha)>0, \text { for each } \alpha \in-\operatorname{Int}(\partial \tilde{t}(\mathbb{R}) .
$$

A straightforward modification of [JK11, Proof of Theorem 1.2] shows that

$$
\operatorname{dim}_{H}\left(\pi_{\tilde{\Phi}}\left(\tilde{\mathcal{F}}^{*}(\alpha)\right)\right)=\operatorname{dim}_{H}\left(\pi_{\tilde{\Phi}}(\tilde{\mathcal{F}}(\alpha))\right)=-\tilde{t}^{*}(-\alpha)
$$


for each $\alpha \in-\operatorname{Int}(\partial \tilde{t}(\mathbb{R})$. The crucial step of this modification is to show that the function $\tilde{t}$ satisfies the exhaustion principle, that is, $\tilde{t}(\beta)=\sup _{n \in \mathbb{N}} \tilde{t}_{n}(\beta)$, where $\tilde{t}_{n}$ is for each $n \in \mathbb{N}$ and $\beta \in \mathbb{R}$ given by

$$
\tilde{t}_{n}(\beta):=\inf \left\{u \in \mathbb{R}: \sum_{k \in \mathbb{N}} \sum_{\left(\tilde{\omega}_{1}, \ldots, \tilde{\omega}_{k}\right) \in \sum_{\tilde{\Phi}}^{k}:\left|\iota\left(\tilde{\omega}_{i}\right)\right| \leq n, 1 \leq i \leq k} \mathrm{e}^{\beta S_{\tilde{\omega}} \tilde{\psi}+u S_{\tilde{\omega}} \tilde{\zeta}}<\infty\right\} .
$$

This exhaustion principle can be verified similarly as in [JK11, Example 1.6, Theorem 1.7] by using that $\Sigma_{\tilde{\Phi}}$ is finitely irreducible (cf. [MU03, Theorem 2.1.5]). Finally, to prove that $-\tilde{t}^{*}(-\alpha)>0$, we observe that $-\tilde{t}^{*}(-\alpha) \geq-\tilde{t}_{n}^{*}(-\alpha)>0$ for all $n$ sufficiently large, which follows from the well-known facts that $-\tilde{t}_{n}^{*}$ is a non-negative, strictly concave and real-analytic function on $\partial \tilde{t}_{n}(\mathbb{R})($ PW97] $)$ and that $\alpha \in-\operatorname{Int}\left(\partial \tilde{t}_{n}(\mathbb{R})(\underline{\text { Roc70 }}\right.$, Theorem 24.5]), for $n$ sufficiently large. The proof is complete.

Recall that the GDMS $\tilde{\Phi}$ is called regular ([MU03, Section 4, p.78]) if there exists $u \in \mathbb{R}$ such that $\mathcal{P}(u \tilde{\zeta})=0$.

Lemma 3.14. Let $\Phi$ denote a conformal GDMS associated with $F_{d}, d \geq 2$, and let $N$ denote a non-trivial normal subgroup of $F_{d}$. Let $\psi: \Sigma_{\Phi} \rightarrow \mathbb{R}$ be Hölder continuous and let $\beta \in \mathbb{R}$. Then $(N, \Phi, \psi)$ is of divergence type in $\beta$ if and only if there exists $u \in \mathbb{R}$ such that $\mathcal{P}(\beta \tilde{\psi}+u \tilde{\zeta})=0$. Moreover, if $N$ is finitely generated, then $(N, \Phi, \psi)$ is of divergence type in $\beta$.

Proof. Suppose that $(N, \Phi, \psi)$ is of divergence type in $\beta$. Then we have $\mathcal{P}(\beta \tilde{\psi}+$ $\left.t_{N}(\beta) \tilde{\zeta}\right) \geq 0$. Further, by Fact 2.3 , we have that $t_{N}(\beta)=\inf \{u \in \mathbb{R}: \mathcal{P}(\beta \tilde{\psi}+$ $u \tilde{\zeta}) \leq 0\}$, because $\Sigma_{\tilde{\Phi}}$ is finitely irreducible by Lemma 3.12 (1). Using again that $\Sigma_{\tilde{\Phi}}$ is finitely irreducible, it follows from [MU03, Theorem 2.1.5] that the map $u \mapsto \mathcal{P}(\beta \tilde{\psi}+u \tilde{\zeta}) \in \mathbb{R} \cup\{\infty\}$ is the monotone limit of a sequence of continuous functions. Consequently, the map $u \mapsto \mathcal{P}(\beta \tilde{\psi}+u \tilde{\zeta})$ is lower semi-continuous, which then implies that $\mathcal{P}\left(\beta \tilde{\psi}+t_{N}(\beta) \tilde{\zeta}\right) \leq 0$. Hence, we have $\mathcal{P}\left(\beta \tilde{\psi}+t_{N}(\beta) \tilde{\zeta}\right)=0$.

To prove the converse, suppose that $\mathcal{P}\left(\beta \tilde{\psi}+t_{N}(\beta) \tilde{\zeta}\right)=0$. Since $\Sigma_{\tilde{\Phi}}$ is finitely irreducible, there exists a Gibbs measure $\mu$ for the potential $\beta \tilde{\psi}+t_{N}(\beta) \tilde{\zeta}$ supported on $\Sigma_{\tilde{\Phi}}$ by Theorem 2.5. Hence, we have that $\sum_{n \in \mathbb{N}} \sum_{\tilde{\omega} \in \Sigma_{\tilde{\Phi}}^{n}} \mu([\tilde{\omega}])=\infty$. Since $\mathcal{P}\left(\beta \tilde{\psi}+t_{N}(\beta) \tilde{\zeta}\right)=0$, it follows from the Gibbs property of $\mu$ (2.1) that $(N, \Phi, \psi)$ is of divergence type in $\beta$.

To finish the proof, suppose that $N$ is finitely generated. Then the edge set of $\tilde{\Phi}$ is finite, and hence, $\tilde{\Phi}$ is regular. In particular, we have that $(N, \Phi, \psi)$ is of divergence type in $\beta$. The proof is complete.

\section{Group-extended Markov systems}

Throughout this section, let $I$ denote a finite or countable alphabet and let $I^{*}$ denote the free semigroup generated by $I$. Let $G$ denote a countable group $G$ and let $\Psi: I^{*} \rightarrow G$ denote a semigroup homomorphism. The skew product dynamical system $\sigma \rtimes \Psi: \Sigma \times G \rightarrow \Sigma \times G$, which is given by

$$
(\sigma \rtimes \Psi)(\tau, g):=\left(\sigma(\tau), g \Psi\left(\tau_{1}\right)\right), \text { for all }(\tau, g) \in \Sigma \times G,
$$


is called a group-extended Markov system (see also [Jae14a, Section 4]). Note that $(\Sigma \times G, \sigma \rtimes \Psi)$ is conjugated to the Markov shift with state space

$$
\left\{\left(\left(\tau_{j}, g_{j}\right)\right) \in(I \times G)^{\mathbb{N}}:\left(\tau_{j}\right) \in \Sigma, \forall i \in \mathbb{N} g_{i} \Psi\left(\tau_{i}\right)=g_{i+1}\right\} .
$$

Let $\pi_{1}: \Sigma \times G \rightarrow \Sigma$ denote the canonical projection.

We will make use of the following notions of symmetry for group-extended Markov systems.

Definition 4.1. Let $(\Sigma \times G, \sigma \rtimes \Psi)$ denote an irreducible group-extended Markov system. We say that $\varphi$ is asymptotically symmetric with respect to $\Psi$ (Jae14a, Definition 3.14]) if there exist $n_{0} \in \mathbb{N}$ and sequences $\left(c_{n}\right) \in\left(\mathbb{R}^{+}\right)^{\mathbb{N}}$ and $\left(N_{n}\right) \in \mathbb{N}^{\mathbb{N}}$ with $\lim _{n}\left(c_{n}\right)^{1 / n}=1$ and $\lim _{n} n^{-1} N_{n}=0$, such that for each $g \in G$ and for all $n \geq n_{0}$,

$$
\sum_{\omega \in \Sigma^{n} \cap \Psi^{-1}(g)} \mathrm{e}^{S_{\omega} \varphi} \leq c_{n} \sum_{\omega \in \Sigma^{*} \cap \Psi^{-1}\left(g^{-1}\right): n-N_{n} \leq|\omega| \leq n+N_{n}} \mathrm{e}^{S_{\omega} \varphi} .
$$

If $\left(c_{n}\right)$ can be chosen to be bounded, then $\varphi$ is called symmetric with respect to $\Psi$. Moreover, $\varphi$ is symmetric on average with respect to $\Psi$ ([Jae12, Definition 1.4]) if

$$
\sup _{g \in G} \limsup _{n \rightarrow \infty} \frac{\sum_{k=1}^{n} \mathrm{e}^{-k p \mathcal{P}\left(\varphi, \Psi^{-1}(\mathrm{id}) \cap \Sigma^{*}\right)} \sum_{\omega \in \Sigma^{k p} \cap \Psi^{-1}(g)} \mathrm{e}^{S_{\omega \varphi}}}{\sum_{k=1}^{n} \mathrm{e}^{-k p \mathcal{P}\left(\varphi, \Psi^{-1}(\mathrm{id}) \cap \Sigma^{*}\right)} \sum_{\omega \in \Sigma^{k p} \cap \Psi^{-1}\left(g^{-1}\right)} \mathrm{e}^{S_{\omega \varphi}}}<\infty,
$$

where $p:=\operatorname{gcd}\left\{n \in \mathbb{N}: \exists \omega \in \Sigma^{n} \cap \Psi^{-1}\right.$ (id) such that $\left.\omega_{n} \omega_{1} \in \Sigma^{2}\right\}$.

Remark 4.2. Throughout this section, we make use of the induced pressure $\mathcal{P}\left(\varphi, \Psi^{-1}\right.$ (id) $\left.\cap \Sigma^{*}\right)$ (see Definition 2.2), where $\Sigma$ is finitely primitive, $(\Sigma \times G, \sigma \rtimes \Psi)$ is an irreducible group-extended Markov system and $\varphi: \Sigma \rightarrow \mathbb{R}$ is Hölder continuous. A straightforward generalisation of the proof of [Jae11, Remark 5.1.6] shows that $\mathcal{P}\left(\varphi, \Psi^{-1}(\mathrm{id}) \cap \Sigma^{*}\right)$ coincides with the Gurevič pressure of $\varphi \circ \pi_{1}$ with respect to $(\Sigma \times G, \sigma \rtimes \Psi)$ (see Sar99]).

4.1. Amenability. Let us first recall the definition of the important property of groups which was introduced by von Neumann Neu29. under the German name messbar. By Day (Day49), groups with this property were renamed amenable groups.

Definition 4.3. A discrete group $G$ is amenable if there exists a finitely additive probability measure $\nu$ on the power set of $G$, such that $\nu(A)=\nu(g(A))$, for all $g \in G$ and $A \subset G$.

The following result is taken from [Jae14b, Corollary 1.6]. (See also [Jae11, Theorem 5.3.11] and [Jae14a, Corollary 4.22 and Remark 4.23], where the case of a finite alphabet was considered.) Stadlbauer proved a similar result for weakly symmetric potentials ([Sta13, Theorem 4.1]).

Theorem 4.4 ([Jae14b, Corollary 1.6]). Let $\Sigma$ be finitely primitive and let $(\Sigma \times G$, $\sigma \rtimes \Psi)$ be an irreducible group-extended Markov system. Suppose that $\varphi: \Sigma \rightarrow \mathbb{R}$ is Hölder continuous with $\mathcal{P}(\varphi)<\infty$ and that $\varphi$ is asymptotically symmetric with respect to $\Psi$. If $G$ is amenable, then $\mathcal{P}\left(\varphi, \Psi^{-1}(\mathrm{id}) \cap \Sigma^{*}\right)=\mathcal{P}(\varphi)$.

The next theorem provides a converse of the previous theorem and is due to Stadlbauer. 
Theorem 4.5 ([Sta13, Theorem 5.4]). Let $\Sigma$ be finitely primitive and let $(\Sigma \times G$, $\sigma \rtimes \Psi)$ be an irreducible group-extended Markov system. Let $\varphi: \Sigma \rightarrow \mathbb{R}$ be Hölder continuous. If $\mathcal{P}\left(\varphi, \Psi^{-1}\right.$ (id) $\left.\cap \Sigma^{*}\right)=\mathcal{P}(\varphi)<\infty$, then $G$ is amenable.

4.2. Recurrence and lower bounds for pressure. Let $\Sigma$ be finitely primitive and let $(\Sigma \times G, \sigma \rtimes \Psi)$ denote an irreducible group-extended Markov system. Let $\varphi: \Sigma \rightarrow \mathbb{R}$ be Hölder continuous with $\mathcal{P}\left(\varphi, \Psi^{-1}\right.$ (id) $\left.\cap \Sigma^{*}\right)<\infty$. The potential $\varphi \circ \pi_{1}: \Sigma \times G \rightarrow \mathbb{R}$ is called recurrent if

$$
\sum_{n \in \mathbb{N}} \mathrm{e}^{-n \mathcal{P}\left(\varphi, \Psi^{-1}(\mathrm{id}) \cap \Sigma^{*}\right)} \sum_{\omega \in \Sigma^{n} \cap \Psi^{-1}(\mathrm{id})} \mathrm{e}^{S_{\omega} \varphi}=\infty .
$$

Remark. It follows from Remark 4.2 that this definition of a recurrent potential coincides with Sarig's definition of a recurrent potential ([Sar01, Definition 1]).

In order to give lower bounds on $\mathcal{P}\left(\varphi, \Psi^{-1}\right.$ (id) $\left.\cap \Sigma^{*}\right)$, we need the following theorem.

Theorem 4.6 ([Jae12, Corollary 1.2, Remark 1.6]). Let $\Sigma$ be finitely primitive and let $(\Sigma \times G, \sigma \rtimes \Psi)$ be an irreducible group-extended Markov system. Let $\varphi: \Sigma \rightarrow \mathbb{R}$ be Hölder continuous with $\mathcal{P}\left(\varphi, \Psi^{-1}(\mathrm{id}) \cap \Sigma^{*}\right)<\infty$. If $\varphi \circ \pi_{1}$ is recurrent, then $G$ is amenable.

For a recurrent potential, we can characterise when $\mathcal{P}\left(\varphi, \Psi^{-1}(\mathrm{id}) \cap \Sigma^{*}\right)$ and $\mathcal{P}(\varphi)$ coincide.

Proposition 4.7 ([Jae12, Proposition 1.5, Remark 1.6]). Let $\Sigma$ be finitely primitive and let $(\Sigma \times G, \sigma \rtimes \Psi)$ be an irreducible group-extended Markov system. Let $\varphi$ : $\Sigma \rightarrow \mathbb{R}$ be Hölder continuous with $\mathcal{P}\left(\varphi, \Psi^{-1}(\mathrm{id}) \cap \Sigma^{*}\right)<\infty$. If $\varphi \circ \pi_{1}$ is recurrent, then we have that $\mathcal{P}\left(\varphi, \Psi^{-1}(\mathrm{id}) \cap \Sigma^{*}\right)=\mathcal{P}(\varphi)$ if and only if $\varphi$ is symmetric on average with respect to $\Psi$.

Remark. By combining Theorem4.6. Theorem 4.4 and Proposition4.7, we see that, if $\varphi \circ \pi_{1}$ is recurrent and if $\varphi$ is asymptotically symmetric with respect to $\Psi$, then $\varphi$ is symmetric on average with respect to $\Psi$.

The next result gives a lower bound on $\mathcal{P}\left(\varphi, \Psi^{-1}(\mathrm{id}) \cap \Sigma^{*}\right)$. A similar result to the first assertion is given in the author's thesis ([Jae11, Theorem 5.3.11]). The second assertion makes use of Theorem 4.6 and is inspired by [Jae14a, Lemma 5.1], where a locally constant potential $\varphi$ is considered.

Proposition 4.8. Let $\Sigma$ be finitely primitive and let $(\Sigma \times G, \sigma \rtimes \Psi)$ be an irreducible group-extended Markov system. For each Hölder continuous potential $\varphi: \Sigma \rightarrow \mathbb{R}$ the following holds.

(1) If $\varphi$ is asymptotically symmetric with respect to $\Psi$, then

$$
2 \mathcal{P}\left(\varphi, \Psi^{-1}(\text { id }) \cap \Sigma^{*}\right) \geq \mathcal{P}(2 \varphi) .
$$

(2) If $\varphi$ is symmetric with respect to $\Psi$ and $\mathcal{P}(2 \varphi)<\infty$, then we have that $2 \mathcal{P}\left(\varphi, \Psi^{-1}(\mathrm{id}) \cap \Sigma^{*}\right)=\mathcal{P}(2 \varphi)$ if and only if $2 \mathcal{P}(\varphi)=\mathcal{P}(2 \varphi)$. 
Proof. We first prove (11). Since $\Sigma$ is finitely primitive and $(\Sigma \times G, \sigma \rtimes \Psi)$ is irreducible, there exists a finite set $B \subset \Psi^{-1}$ (id) $\cap \Sigma^{*}$ such that, for all $\omega_{1}, \omega_{2} \in \Sigma^{*}$ there is $\gamma\left(\omega_{1}, \omega_{2}\right) \in B$ with $\omega_{1} \gamma\left(\omega_{1}, \omega_{2}\right) \omega_{2} \in \Sigma^{*}$. Define the map $\Gamma: \Sigma^{*} \times \Sigma^{*} \rightarrow \Sigma^{*}$ given by $\Gamma\left(\omega_{1}, \omega_{2}\right):=\omega_{1} \gamma\left(\omega_{1}, \omega_{2}\right) \omega_{2}$, where $\gamma\left(\omega_{1}, \omega_{2}\right) \in B$. Note that the restriction of $\Gamma$ to $\Sigma^{n} \times \Sigma^{*}$ (resp. $\left.\Sigma^{*} \times \Sigma^{n}\right)$ is at most card $(B)$-to-one, for each $n \in \mathbb{N}$. Setting $C_{B}:=\min \left\{S_{\gamma} \varphi: \gamma \in B\right\}>-\infty$ and using the bounded distortion property of $\varphi$ with constant $C_{\varphi}>0$ (see Fact 2.1), we have that $S_{\omega_{1}} \varphi+S_{\omega_{2}} \varphi-3 C_{\varphi}+C_{B} \leq$ $S_{\Gamma\left(\omega_{1}, \omega_{2}\right)} \varphi$, for all $\omega_{1}, \omega_{2} \in \Sigma^{*}$. Consequently, setting $l:=\max \{|\gamma|: \gamma \in B\}$, we obtain for every sequence $\left(N_{n}\right) \in \mathbb{N}^{\mathbb{N}}$ and $n \in \mathbb{N}$,

$$
\begin{aligned}
& \operatorname{card}(B) \mathrm{e}^{3 C_{\varphi}-C_{B}} \sum_{\omega \in \Sigma^{*} \cap \Psi^{-1}(\mathrm{id}): 2 n-N_{n} \leq|\omega| \leq 2 n+N_{n}+l} \mathrm{e}^{S_{\omega} \varphi} \sum_{g \in G}\left(\sum_{\omega_{1} \in \Sigma^{n} \cap \Psi^{-1}(g)} \mathrm{e}^{S_{\omega_{1}} \varphi}\right)\left(\sum_{\omega_{2} \in \Sigma^{*} \cap \Psi^{-1}\left(g^{-1}\right): n-N_{n} \leq\left|\omega_{2}\right| \leq n+N_{n}} \mathrm{e}^{S_{\omega_{2}} \varphi}\right) .
\end{aligned}
$$

Using that $\varphi$ is asymptotically symmetric with respect to $\Psi$ with $n_{0} \in \mathbb{N}$ and sequences $\left(c_{n}\right) \in \mathbb{R}^{\mathbb{N}}$ and $\left(N_{n}\right) \in \mathbb{N}^{\mathbb{N}}$ as in Definition 4.1 it follows from the previous inequality that for all $n \geq n_{0}$,

$$
\begin{aligned}
\operatorname{card} & (B) \mathrm{e}^{3 C_{\varphi}-C_{B}} c_{n} \sum_{\omega \in \Sigma^{*} \cap \Psi^{-1}(\mathrm{id}): 2 n-N_{n} \leq|\omega| \leq 2 n+N_{n}+l} \mathrm{e}^{S_{\omega} \varphi} \\
& \geq \sum_{g \in G}\left(\sum_{\omega_{1} \in \Sigma^{n} \cap \Psi^{-1}(g)} \mathrm{e}^{S_{\omega_{1}} \varphi}\right)\left(\sum_{\omega_{2} \in \Sigma^{n} \cap \Psi^{-1}(g)} \mathrm{e}^{S_{\omega_{2}} \varphi}\right) \\
& \geq \sum_{g \in G}\left(\sum_{\omega_{1} \in \Sigma^{n} \cap \Psi^{-1}(g)} \mathrm{e}^{2 S_{\omega_{1}} \varphi}\right)=\sum_{\omega \in \Sigma^{n}} \mathrm{e}^{2 S_{\omega} \varphi} .
\end{aligned}
$$

Using that $\lim _{n}\left(c_{n}\right)^{1 / n}=1$ it follows from (4.1) that

$$
\begin{gathered}
\limsup _{n \rightarrow \infty} \frac{1}{n} \sum_{\omega \in \Sigma^{*} \cap \Psi^{-1}(\mathrm{id}): 2 n-N_{n} \leq|\omega| \leq 2 n+N_{n}+l} \mathrm{e}^{S_{\omega \varphi}} \\
\geq \limsup _{n \rightarrow \infty} \frac{1}{n} \log \sum_{\omega \in \Sigma^{n}} \mathrm{e}^{2 S_{\omega} \varphi}=\mathcal{P}(2 \varphi) .
\end{gathered}
$$

Finally, using that $\lim _{n} n^{-1} N_{n}=0$, one verifies that

$$
2 \mathcal{P}\left(\varphi, \Psi^{-1}\{\mathrm{id}\} \cap \Sigma^{*}\right) \geq \limsup _{n \rightarrow \infty} \frac{1}{n} \log \sum_{\omega \in \Sigma^{*} \cap \Psi^{-1}(\mathrm{id}): 2 n-N_{n} \leq|\omega| \leq 2 n+N_{n}+l} \mathrm{e}^{S_{\omega} \varphi},
$$

which finishes the proof of (11).

We now turn to the proof of (2). First, note that by passing to the potential $\varphi-\mathcal{P}(2 \varphi) / 2$, we may assume without loss of generality that $\mathcal{P}(2 \varphi)=0$. It remains to show that $\mathcal{P}\left(\varphi, \Psi^{-1}(\right.$ id $\left.) \cap \Sigma^{*}\right)=0$ if and only if $\mathcal{P}(\varphi)=0$. Since $\mathcal{P}\left(\varphi, \Psi^{-1}(\mathrm{id}) \cap \Sigma^{*}\right) \geq \mathcal{P}(2 \varphi) / 2=0$ by (11), we deduce that $\mathcal{P}(\varphi)=0$ implies $\mathcal{P}\left(\varphi, \Psi^{-1}(\mathrm{id}) \cap \Sigma^{*}\right)=0$. Now, for the opposite implication, suppose that $\mathcal{P}\left(\varphi, \Psi^{-1}(\mathrm{id}) \cap \Sigma^{*}\right)=0$. Since $\Sigma$ is finitely primitive and $\mathcal{P}(2 \varphi)=0$ there exists a unique $\sigma$-invariant Gibbs measure $\mu$ for $2 \varphi$ by Theorem 2.5. By (2.1) there exists a constant $C_{\mu}>0$ such that for all $n \in \mathbb{N}$ we have

$$
\sum_{\omega \in \Sigma^{n}} \mathrm{e}^{2 S_{\omega} \varphi} \geq C_{\mu}^{-1} \sum_{\omega \in \Sigma^{n}} \mu([\omega])=C_{\mu}^{-1}>0
$$


Since $\varphi$ is symmetric with respect to $\Psi$ and by (4.1), there exists $n_{0} \in \mathbb{N}$ and $C>0$, such that for all $n \geq n_{0} \in \mathbb{N}$,

$$
\sum_{\omega \in \Sigma^{*} \cap \Psi^{-1} \text { (id):2n-N } \leq|\omega| \leq 2 n+N_{n}+l} \mathrm{e}^{S_{\omega} \varphi} \geq C \sum_{\omega \in \Sigma^{n}} \mathrm{e}^{2 S_{\omega} \varphi} \geq C C_{\mu}^{-1}>0 .
$$

Since $\lim _{n} n^{-1} N_{n}=0$, there exists a sequence $\left(n_{k}\right) \in \mathbb{N}^{\mathbb{N}}$ tending to infinity, such that the sets $\left\{2 n_{k}-N_{n_{k}}, \ldots, 2 n_{k}+N_{n_{k}}+l\right\}_{k \in \mathbb{N}}$ are pairwise disjoint. Hence, by (4.2), we have that

$$
\sum_{n \in \mathbb{N}} \sum_{\omega \in \Sigma^{n} \cap \Psi^{-1}(\mathrm{id})} \mathrm{e}^{S_{\omega} \varphi}=\infty
$$

Since $\mathcal{P}\left(\varphi, \Psi^{-1}(\mathrm{id}) \cap \Sigma^{*}\right)=0$, we have thus shown that $\varphi \circ \pi_{1}$ is a recurrent. Hence, Theorem 4.6 gives that $G$ is amenable. Finally, it follows from Theorem 4.4 that $0=\mathcal{P}\left(\varphi, \Psi^{-1}\right.$ (id) $\left.\cap \Sigma^{*}\right)=\mathcal{P}(\varphi)$, which finishes the proof of (2).

Corollary 4.9. Let $\Sigma$ be finitely primitive and let $(\Sigma \times G, \sigma \rtimes \Psi)$ be an irreducible group-extended Markov system. Let $\varphi: \Sigma \rightarrow \mathbb{R}$ be Hölder continuous with $\mathcal{P}(2 \varphi)<$ $\infty$ and suppose that $\varphi$ is symmetric with respect to $\Psi$. If $G$ is non-amenable, then $2 \mathcal{P}\left(\varphi, \Psi^{-1}(\mathrm{id}) \cap \Sigma^{*}\right)>\mathcal{P}(2 \varphi)$.

Proof. Suppose for a contradiction that the claim is false. Then, by Proposition 4.8 (1) and (2), we have $2 \mathcal{P}\left(\varphi, \Psi^{-1}(\right.$ id $\left.) \cap \Sigma^{*}\right)=\mathcal{P}(2 \varphi)=2 \mathcal{P}(\varphi)$. By Theorem 4.5 we conclude that $G$ is amenable, which is a contradiction.

\section{Proof of the Main Results}

For a conformal GDMS $\Phi$ associated with $F_{d}=\left\langle g_{1}, \ldots, g_{d}\right\rangle, d \geq 2$, set $I:=$ $\left\{g_{1}, g_{1}^{-1}, \ldots, g_{d}, g_{d}^{-1}\right\}$ and $\Sigma:=\left\{\tau \in I^{\mathbb{N}}: \forall i \in \mathbb{N} \tau_{i} \neq \tau_{i+1}^{-1}\right\}$. One immediately verifies that the Markov shift $\Sigma$ is finitely primitive. For a non-trivial normal subgroup $N$ of $F_{d}$, let $\Psi_{N}: I^{*} \rightarrow F_{d} / N$ denote the canonical semigroup homomorphism given by $\Psi_{N}(g):=N g$, for each $g \in I$. Using that $d \geq 2$ and that $N$ is a non-trivial normal subgroup of $F_{d}$, we see that the group-extended Markov system $\sigma \rtimes \Psi_{N}: \Sigma \times\left(F_{d} / N\right) \rightarrow \Sigma \times\left(F_{d} / N\right)$ is irreducible. We consider $N \backslash\{$ id $\}$ as a subset of $\Sigma^{*}$. To apply the results of Section 4 , we will frequently make use of the fact that

$$
N \backslash\{\mathrm{id}\}=\Psi_{N}^{-1}(\mathrm{id}) \cap \Sigma^{*} .
$$

The geometric potential $\zeta$ of $\Phi$ is Hölder continuous by Fact 3.5. Since the Lipschitz constants of $\Phi$ are bounded away from one, we have that $\sup _{\tau \in \Sigma} \zeta(\tau)<0$. Since $\operatorname{card}(I)<\infty$ we have the following by Fact 2.3 .

Fact 5.1. Let $\psi: \Sigma \rightarrow \mathbb{R}$ be Hölder continuous and let $t_{N}: \mathbb{R} \rightarrow \mathbb{R}$ denote the free energy function of $(N, \Phi, \psi)$. For each $\beta \in \mathbb{R}$, we have that $t_{N}(\beta)=$ $\mathcal{P}_{-\zeta}(\beta \psi, N \backslash\{\mathrm{id}\})$, the function $u \mapsto \mathcal{P}(\beta \psi+u \zeta, N \backslash\{\mathrm{id}\})$ is strictly decreasing with values in $\mathbb{R}$ and $\mathcal{P}\left(\beta \psi+t_{N}(\beta) \zeta, N \backslash\{\operatorname{id}\}\right)=0$.

Lemma 5.2. We have $\delta_{N}>0$.

Proof. Since $0: \Sigma \rightarrow\{0\}$ is asymptotically symmetric with respect to $\Psi_{N}$, we have

$$
2 \mathcal{P}(0, N \backslash\{\operatorname{id}\}) \geq \mathcal{P}(0)=\log (2 d-1)>0
$$

by Proposition 4.8 (1). Hence, we have $\delta_{N}=t_{N}(0)>0$ by Fact 5.1 . 
We will repeatedly make use of the following fact about the convex conjugate of a convex function. For the proof we refer to [Roc70, Theorem 23.5].

Fact 5.3. Let $f: \mathbb{R} \rightarrow \mathbb{R}$ be a convex function and let $f^{*}: \mathbb{R} \rightarrow \mathbb{R} \cup\{\infty\}$ denote the convex conjugate of $f$.

(1) Let $\beta \in \mathbb{R}$. If $\alpha \in \partial f(\beta)$, then $f^{*}(\alpha)=\alpha \beta-f(\beta)$.

(2) $\partial f(\mathbb{R}) \subset\left\{x \in \mathbb{R}: f^{*}(x)<\infty\right\} \subset \overline{\partial f(\mathbb{R})}$.

The following results about the free energy function are crucial to derive Theorems 1.1 and 1.2 .

Proposition 5.4. Let $\Phi$ denote a conformal GDMS associated with $F_{d}, d \geq 2$, and let $N$ denote a non-trivial normal subgroup of $F_{d}$. Let $\psi: \Sigma \rightarrow \mathbb{R}$ be Hölder continuous and let $t_{N}: \mathbb{R} \rightarrow \mathbb{R}$ and $t: \mathbb{R} \rightarrow \mathbb{R}$ denote the free energy functions of $(N, \Phi, \psi)$ and $\left(F_{d}, \Phi, \psi\right)$ respectively. Then we have the following.

(1) (a) We have $t_{N}(\beta) \leq t(\beta)$ and $-t_{N}^{*}(-\alpha) \leq-t^{*}(-\alpha)$ for all $\beta, \alpha \in \mathbb{R}$.

(b) If $t_{N}(\beta)=t(\beta)$ for some $\beta \in \mathbb{R}$, then $F_{d} / N$ is amenable.

(c) If $-t_{N}^{*}(-\alpha)=-t^{*}(-\alpha)$ for some $\alpha \in-\partial t(\mathbb{R})$, then $F_{d} / N$ is amenable.

(d) $\operatorname{Int}\left(\partial t_{N}(\mathbb{R})\right) \subset \operatorname{Int}(\partial t(\mathbb{R}))$.

(2) Suppose that $(N, \Phi, \psi)$ is asymptotically symmetric.

(a) If $F_{d} / N$ is amenable, then $t_{N}(\beta)=t(\beta)$ and $-t_{N}^{*}(-\alpha)=-t^{*}(-\alpha)$ for all $\beta, \alpha \in \mathbb{R}$.

(b) We have $2 t_{N}(\beta) \geq t(2 \beta)$ and $-t_{N}^{*}(-\alpha) \geq-t^{*}(-\alpha) / 2$ for all $\beta, \alpha \in$ $\mathbb{R}$.

(c) If $(N, \Phi, \psi)$ is symmetric and $F_{d} / N$ is non-amenable, then we have $2 t_{N}(\beta)>t(2 \beta)$ and $-t_{N}^{*}(-\alpha)>-t^{*}(-\alpha) / 2$, for every $\beta \in \mathbb{R}$ and $\alpha \in-\partial t_{N}(\mathbb{R})$.

(d) $\operatorname{Int}\left(\partial t_{N}(\mathbb{R})\right)=\operatorname{Int}(\partial t(\mathbb{R}))$.

(3) Let $\beta \in \mathbb{R}$ and suppose that $(N, \Phi, \psi)$ is of divergence type in $\beta$.

(a) Then $F_{d} / N$ is amenable.

(b) $t_{N}(\beta)=t(\beta)$ if and only if $(N, \Phi, \psi)$ is symmetric on average in $\beta$.

(c) If $\alpha \in-\partial t(\beta)$ and $-t_{N}^{*}(-\alpha)=-t^{*}(-\alpha)$, then $(N, \Phi, \psi)$ is symmetric on average in $\beta$.

(d) If $(N, \Phi, \psi)$ is symmetric on average in $\beta$, then $-t_{N}^{*}(-\alpha)=-t^{*}(-\alpha)$ for each $\alpha \in-\partial t_{N}(\beta)$.

Proof. The first assertion in (1a) follows from Fact [5.1, since we have

$$
\mathcal{P}(\beta \psi+t(\beta) \zeta, N \backslash\{\operatorname{id}\}) \leq \mathcal{P}(\beta \psi+t(\beta) \zeta)=0, \text { for } \beta \in \mathbb{R} .
$$

By the definition of the convex conjugate, we then have for $\alpha \in \mathbb{R}$,

$$
-t_{N}^{*}(-\alpha)=\inf _{\beta \in \mathbb{R}}\left\{t_{N}(\beta)+\beta \alpha\right\} \leq \inf _{\beta \in \mathbb{R}}\{t(\beta)+\beta \alpha\}=-t^{*}(-\alpha),
$$

which proves the second assertion in (1a). To prove (1b), suppose that $t_{N}(\beta)=t(\beta)$ for some $\beta \in \mathbb{R}$. Then by Fact 5.1 we have that $\mathcal{P}\left(\beta \psi+t_{N}(\beta) \zeta, N \backslash\{\operatorname{id}\}\right)=$ $\mathcal{P}(\beta \psi+t(\beta) \zeta)=0$. Applying Theorem 4.5 to the Hölder continuous potential $\beta \psi+t_{N}(\beta) \zeta: \Sigma \rightarrow \mathbb{R}$ and the group-extended Markov system $\left(\Sigma \times\left(F_{d} / N\right), \sigma \rtimes \Psi_{N}\right)$ gives that $F_{d} / N$ is amenable. For the proof of (1c), let $\alpha \in-\partial t(\beta)$ for some $\beta \in \mathbb{R}$. 
By Fact 5.3 (1), the first inequality in (1a) and the definition of the convex conjugate, we have

$$
-t^{*}(-\alpha)=t(\beta)+\beta \alpha \geq t_{N}(\beta)+\beta \alpha \geq-t_{N}^{*}(-\alpha) .
$$

Consequently, if $-t_{N}^{*}(-\alpha)=-t^{*}(-\alpha)$, then we have $t_{N}(\beta)=t(\beta)$ and amenability of $F_{d} / N$ follows from (1b). To prove (1d), let $\alpha \in \operatorname{Int}\left(\partial t_{N}(\mathbb{R})\right)$. By Fact $5.3(2)$ we have $t_{N}^{*}(\alpha)<\infty$. By (1a) we have $t^{*}(\alpha) \leq t_{N}^{*}(\alpha)<\infty$. By Fact 5.3 (2) again, we conclude that $\alpha \in \overline{\partial t(\mathbb{R})}$. Since $\alpha$ is an interior point of $\partial t_{N}(\mathbb{R})$, we have thus shown that $\operatorname{Int}\left(\partial t_{N}(\mathbb{R})\right) \subset \operatorname{Int}(\partial t(\mathbb{R}))$.

Now suppose that $(N, \Phi, \psi)$ is asymptotically symmetric, that is, $\beta \psi+u \zeta$ is asymptotically symmetric with respect to $\Psi_{N}$, for all $\beta, u \in \mathbb{R}$. To prove (2a), suppose that $F_{d} / N$ is amenable. By applying Theorem 4.4 to the Hölder continuous potential $\beta \psi+t(\beta) \zeta$ and the group-extended Markov system $\left(\Sigma \times\left(F_{d} / N\right), \sigma \rtimes \Psi_{N}\right)$, we obtain by Fact 5.1 that $\mathcal{P}(\beta \psi+t(\beta) \zeta, N \backslash\{$ id $\})=\mathcal{P}(\beta \psi+t(\beta) \zeta)=0$ for $\beta \in \mathbb{R}$. Consequently, we have $t_{N}(\beta)=t(\beta)$ for all $\beta \in \mathbb{R}$ by Fact 5.1 and thus, $-t_{N}^{*}(-\alpha)=-t^{*}(-\alpha)$ for all $\alpha \in \mathbb{R}$. To prove (2b) let $\beta \in \mathbb{R}$. Applying Proposition 4.8 (1) to the asymptotically symmetric Hölder continuous potential $\beta \psi+(t(2 \beta) / 2) \zeta$ gives that

$$
2 \mathcal{P}(\beta \psi+(t(2 \beta) / 2) \zeta, N \backslash\{\operatorname{id}\}) \geq \mathcal{P}(2 \beta \psi+t(2 \beta) \zeta)=0 .
$$

Hence, we have $t_{N}(\beta) \geq t(2 \beta) / 2$ by Fact 5.1 and we obtain for $\alpha \in \mathbb{R}$,

$$
-t_{N}^{*}(-\alpha)=\inf _{\beta \in \mathbb{R}}\left\{t_{N}(\beta)+\beta \alpha\right\} \geq \inf _{\beta \in \mathbb{R}}\{t(2 \beta)+2 \beta \alpha\} / 2=-t^{*}(-\alpha) / 2,
$$

which finishes the proof of (2b). To prove (2c) suppose that $(N, \Phi, \psi)$ is symmetric and that $F_{d} / N$ is non-amenable. Then the inequality in (5.1) is strict by Corollary 4.9. Hence, $t_{N}(\beta)>t(2 \beta) / 2$ for every $\beta \in \mathbb{R}$. Moreover, if $\alpha \in-\partial t_{N}(\beta)$ for some $\beta \in \mathbb{R}$, then by using Fact 5.3 (1) we deduce that

$$
-t_{N}^{*}(-\alpha)=t_{N}(\beta)+\beta \alpha>(t(2 \beta)+2 \beta \alpha) / 2 \geq-t^{*}(-\alpha) / 2 .
$$

To prove (2d), it suffices to show that $\partial t(\mathbb{R}) \subset \overline{\partial t_{N}(\mathbb{R})}$. Then $\operatorname{Int}\left(\partial t_{N}(\mathbb{R})\right)=$ $\operatorname{Int}(\partial t(\mathbb{R}))$ follows by combining with (1d $)$. Let $\alpha \in-\partial t(\beta)$ for some $\beta \in \mathbb{R}$. Then by (2b) and Fact 5.3 (1) we have

$$
-t_{N}^{*}(-\alpha) \geq-t^{*}(-\alpha) / 2=(t(\beta)+\beta \alpha) / 2>-\infty,
$$

which shows that $\alpha \in-\overline{\partial t_{N}(\mathbb{R})}$ by Fact 5.3 (2).

To prove (3) let $\beta \in \mathbb{R}$ and suppose that $(N, \Phi, \psi)$ is of divergence type in $\beta$, that is, $\left(\beta \psi+t_{N}(\beta) \zeta\right) \circ \pi_{1}$ is recurrent with respect to $\sigma \rtimes \Psi_{N}$. Then amenability of $F_{d} / N$ follows from Theorem 4.6, which proves (3a). To prove (3b), first observe that $(N, \Phi, \psi)$ is symmetric on average in $\beta$ if and only if $\beta \psi+t(\beta) \zeta$ is symmetric on average with respect to $\Psi_{N}$. Now, the equivalence in (3b) follows from Proposition 4.7 and Fact 5.1] To prove (3c), suppose that $-t_{N}^{*}(-\alpha)=-t^{*}(-\alpha)$ for $\alpha \in-\partial t(\beta)$. As in the proof of (1c) we deduce that $t_{N}(\beta)=t(\beta)$, which then implies that $(N, \Phi, \psi)$ is symmetric on average in $\beta$ by (3b). In order to prove (3d), suppose that $(N, \Phi, \psi)$ is symmetric on average in $\beta$ and let $\alpha \in-\partial t_{N}(\beta)$. By (3b) we then have $t_{N}(\beta)=t(\beta)$. Hence, we obtain by Fact 5.3 (11) that

$$
-t_{N}^{*}(-\alpha)=t_{N}(\beta)+\beta \alpha=t(\beta)+\beta \alpha \geq-t^{*}(-\alpha) .
$$

Combining with (1ad) finishes the proof of (3d) and completes the proof of the proposition. 
We are now in the position to prove the main theorems.

Proof of Theorem 1.1. The first assertion follows from Proposition 3.13 (1) and Lemma 5.2. To apply Proposition 5.4 in what follows, recall that $\delta_{N}=t_{N}(0)$ and $\delta=t(0)$, where $t_{N}: \mathbb{R} \rightarrow \mathbb{R}$ and $t: \mathbb{R} \rightarrow \mathbb{R}$ denote the free energy functions of $(N, \Phi, 0)$ and $\left(F_{d}, \Phi, 0\right)$ respectively. To prove (2), suppose that $F_{d} / N$ is nonamenable. By (1a) and (1b) of Proposition 5.4, we have $\delta_{N}<\delta$. Combining with (11), finishes the proof of (2).

For the proof of (3), suppose that $(N, \Phi, 0)$ is asymptotically symmetric. If $F_{d} / N$ is amenable, then we have $\delta_{N}=\delta$ by Proposition 5.4 (2a). Now suppose that $F_{d} / N$ is non-amenable. Using Proposition 5.4 (2b) we obtain that $\delta_{N} \geq \delta / 2$, and if $(N, \Phi, 0)$ is symmetric, then we have $\delta_{N}>\delta / 2$ by Proposition 5.4 (2c). In light of (11) the proof of (3) is complete.

To prove (44), suppose that $(N, \Phi)$ is of divergence type, that is, $(N, \Phi, 0)$ is of divergence type in 0. Then the assertion in (4a) follows from Proposition 5.4 (3a) and the assertion in (4b) follows from Proposition 5.4 (3b) for $\beta=0$. The proof is complete.

Proof of Theorem 1.2. The first assertion in (11) follows from Proposition 5.4 (1d) and Proposition 3.13 (3). The second assertion in (1) follows from Proposition 5.4 (2d). To prove (2), suppose that $F_{d} / N$ is non-amenable and let $\alpha \in\left(\alpha_{-}, \alpha_{+}\right)$. Since we have $\left(\alpha_{-}, \alpha_{+}\right)=-\operatorname{Int}(\partial t(\mathbb{R}))$, it follows from (1a) and (1c) of Proposition 5.4 that we have $-t_{N}^{*}(-\alpha)<-t^{*}(-\alpha)$. Further, by Proposition 3.13 (3), we have $\operatorname{dim}_{H}\left(\pi_{\Phi}(\mathcal{F}(\alpha, \Phi, \psi))\right)=-t^{*}(-\alpha)>0$. Consequently, we obtain by Proposition 3.13 (2) that

$$
\begin{aligned}
\operatorname{dim}_{H}\left(\pi_{\Phi}\left(\Lambda_{\mathrm{r}}(N) \cap \mathcal{F}(\alpha, \Phi, \psi)\right)\right) & \leq \max \left\{-t_{N}^{*}(-\alpha), 0\right\}<-t^{*}(-\alpha) \\
& =\operatorname{dim}_{H}\left(\pi_{\Phi}(\mathcal{F}(\alpha, \Phi, \psi))\right),
\end{aligned}
$$

which gives the desired inequality in (2).

Now suppose that $(N, \Phi, \psi)$ is asymptotically symmetric and let $\alpha \in\left(\alpha_{-}, \alpha_{+}\right)$. By Proposition $5.4(2 \mathrm{~d})$, we have $\operatorname{Int}\left(\partial t_{N}(\mathbb{R})\right)=\operatorname{Int}(\partial t(\mathbb{R}))$. Hence, we have $\operatorname{dim}_{H}\left(\pi_{\Phi}\left(\Lambda_{\mathrm{r}}(N) \cap \mathcal{F}(\alpha, \Phi, \psi)\right)\right)=-t_{N}^{*}(-\alpha)$ and $\operatorname{dim}_{H}\left(\pi_{\Phi}(\mathcal{F}(\alpha, \Phi, \psi))\right)$ $=-t^{*}(-\alpha)$ by Proposition 3.13 (3). The assertion in (3a) is then a consequence of Proposition 5.4 (2a). The lower bound in (3b) is deduced from Proposition 5.4 (2b). Finally, if $(N, \Phi, \psi)$ is symmetric, then the strict inequality in (3b) follows from Proposition 5.4 (2c).

Let us now turn to the proof of (4). Let $\beta \in \mathbb{R}$ and suppose that $(N, \Phi, \psi)$ is of divergence type in $\beta$. The assertion in (4a) is proved in Proposition 5.4 (3a). To prove (4b), let $\alpha \in-\partial t(\beta)$ and suppose that $\operatorname{dim}_{H}\left(\pi_{\Phi}\left(\Lambda_{\mathrm{r}}(N) \cap \mathcal{F}(\alpha, \Phi, \psi)\right)\right)=$ $\operatorname{dim}_{H}\left(\pi_{\Phi}(\mathcal{F}(\alpha, \Phi, \psi))\right)$. By Proposition 3.13 (2) and (3) we then have that

$$
\begin{aligned}
0<-t^{*}(-\alpha) & =\operatorname{dim}_{H}\left(\pi_{\Phi}(\mathcal{F}(\alpha, \Phi, \psi))\right)=\operatorname{dim}_{H}\left(\pi_{\Phi}\left(\Lambda_{\mathrm{r}}(N) \cap \mathcal{F}(\alpha, \Phi, \psi)\right)\right) \\
& \leq \max \left\{-t_{N}^{*}(-\alpha), 0\right\} .
\end{aligned}
$$

Hence, we have $-t_{N}^{*}(-\alpha)=-t^{*}(-\alpha)$ and Proposition 5.4 (3c) gives that $(N, \Phi, \psi)$ is symmetric on average in $\beta$. Finally, to prove (4c), let

$$
\alpha \in-\left(\partial t_{N}(\beta) \cap \operatorname{Int}\left(\partial t_{N}(\mathbb{R})\right)\right) .
$$


Suppose that $(N, \Phi, \psi)$ is symmetric on average in $\beta$. Then we have $-t_{N}^{*}(-\alpha)=$ $-t^{*}(-\alpha)$ by Proposition $5.4(3 \mathrm{~d})$. Since $-t^{*}(-\alpha)=\operatorname{dim}_{H}\left(\pi_{\Phi}(\mathcal{F}(\alpha, \Phi, \psi))\right)$ and $-t_{N}^{*}(-\alpha)=\operatorname{dim}_{H}\left(\pi_{\Phi}\left(\Lambda_{\mathrm{r}}(N) \cap \mathcal{F}(\alpha, \Phi, \psi)\right)\right)$ by Proposition 3.13 (3) and Proposition $5.4(1 \mathrm{~d})$, the proof is complete.

\section{REFERENCES}

[Bea95] Alan F. Beardon, The geometry of discrete groups, Graduate Texts in Mathematics, vol. 91, Springer-Verlag, New York, 1995. Corrected reprint of the 1983 original. MR.1393195 (97d:22011)

[BJ97] Christopher J. Bishop and Peter W. Jones, Hausdorff dimension and Kleinian groups, Acta Math. 179 (1997), no. 1, 1-39, DOI 10.1007/BF02392718. MR.1484767 (98k:22043)

[Bow75] Rufus Bowen, Equilibrium states and the ergodic theory of Anosov diffeomorphisms, Lecture Notes in Mathematics, Vol. 470, Springer-Verlag, Berlin, 1975. MR.0442989 (56 \#1364)

[Bro85] Robert Brooks, The bottom of the spectrum of a Riemannian covering, J. Reine Angew. Math. 357 (1985), 101-114, DOI 10.1515/crll.1985.357.101. MR783536 (86h:58138)

[BTMT12] Petra Bonfert-Taylor, Katsuhiko Matsuzaki, and Edward C. Taylor, Large and small covers of a hyperbolic manifold, J. Geom. Anal. 22 (2012), no. 2, 455-470, DOI 10.1007/s12220-010-9204-6. MR2891734

[Coh82] Joel M. Cohen, Cogrowth and amenability of discrete groups, J. Funct. Anal. 48 (1982), no. 3, 301-309, DOI 10.1016/0022-1236(82)90090-8. MR678175 (85e:43004)

[Day49] M. M. Day, Means on semigroups and groups, Bull. Amer. Math. Soc. 55 (1949), 1054-1055.

[FS04] Kurt Falk and Bernd O. Stratmann, Remarks on Hausdorff dimensions for transient limit sets of Kleinian groups, Tohoku Math. J. (2) 56 (2004), no. 4, 571-582. MR2097162 (2005g:30053)

[Gri80] R. I. Grigorchuk, Symmetrical random walks on discrete groups, Multicomponent random systems, Adv. Probab. Related Topics, vol. 6, Dekker, New York, 1980, pp. 285325. MR:599539 (83k:60016)

[Jae11] Johannes Jaerisch, Thermodynamic Formalism for Group-Extended Markov Systems with Applications to Fuchsian Groups, Ph.D. thesis, University Bremen, http://d-nb.info/1011939185/34, 2011.

[Jae12] , Recurrence and pressure for group extensions, preprint available at http://arxiv.org/abs/1205.4490 (2012).

[Jae13] - A lower bound for the exponent of convergence of normal subgroups of Kleinian groups, J. Geom. Anal. (accepted for publication), doi: 10.1007/s12220-013-9427-4 (2013).

[Jae14a] , Fractal models for normal subgroups of Schottky groups, Trans. Amer. Math. Soc. (accepted for publication), preprint available at http://arxiv.org/abs/1106.0026 (2014).

[Jae14b] - Group-extended Markov systems, amenability, and the Perron-Frobenius operator, Proc. Amer. Math. Soc. (accepted for publication), preprint available at http://arxiv.org/abs/1205.5126 (2014).

[JK11] Johannes Jaerisch and Marc Kesseböhmer, Regularity of multifractal spectra of conformal iterated function systems, Trans. Amer. Math. Soc. 363 (2011), no. 1, 313-330, DOI 10.1090/S0002-9947-2010-05326-7. MR2719683(2012b:37064)

[JKL14] Johannes Jaerisch, Marc Kesseböhmer, and Sanaz Lamei, Induced topological pressure for countable state Markov shifts, Stoch. Dyn. 14 (2014), no. 2, (to appear) doi: 10.1142/S0219493713500160.

[KMS12] Marc Kesseböhmer, Sara Munday, and Bernd O. Stratmann, Strong renewal theorems and Lyapunov spectra for $\alpha$-Farey and $\alpha$-Lüroth systems, Ergodic Theory Dynam. Systems 32 (2012), no. 3, 989-1017, DOI 10.1017/S0143385711000186. MR2995653 
[Mas88] Bernard Maskit, Kleinian groups, Grundlehren der Mathematischen Wissenschaften [Fundamental Principles of Mathematical Sciences], vol. 287, Springer-Verlag, Berlin, 1988. MR959135 (90a:30132)

[MT98] Katsuhiko Matsuzaki and Masahiko Taniguchi, Hyperbolic manifolds and Kleinian groups, Oxford Mathematical Monographs, The Clarendon Press Oxford University Press, New York, 1998. Oxford Science Publications. MR.1638795 (99g:30055)

[MU96] R. Daniel Mauldin and Mariusz Urbański, Dimensions and measures in infinite iterated function systems, Proc. London Math. Soc. (3) 73 (1996), no. 1, 105-154, DOI 10.1112/plms/s3-73.1.105. MR.1387085 (97c:28020)

[MU03] R. Daniel Mauldin and Mariusz Urbański, Graph directed Markov systems, Cambridge Tracts in Mathematics, vol. 148, Cambridge, 2003.

[MY09] Katsuhiko Matsuzaki and Yasuhiro Yabuki, The Patterson-Sullivan measure and proper conjugation for Kleinian groups of divergence type, Ergodic Theory Dynam. Systems 29 (2009), no. 2, 657-665, DOI 10.1017/S0143385708080267. MR2486788 (2010h:37097)

[Neu29] J. v. Neumann, Zur allgemeinen Theorie des Masses, Fund. Math. 13 (1929), 73-116.

[Nic89] Peter J. Nicholls, The ergodic theory of discrete groups, London Mathematical Society Lecture Note Series, vol. 143, Cambridge University Press, Cambridge, 1989. MR:1041575 (91i:58104)

[PW97] Yakov Pesin and Howard Weiss, A multifractal analysis of equilibrium measures for conformal expanding maps and Moran-like geometric constructions, J. Statist. Phys. 86 (1997), no. 1-2, 233-275, DOI 10.1007/BF02180206. MR 1435198 (97m:58118)

[Ree81a] Mary Rees, Checking ergodicity of some geodesic flows with infinite Gibbs measure, Ergodic Theory Dynamical Systems 1 (1981), no. 1, 107-133. MR627791 (83g:58037)

[Ree81b] Mary Rees, Divergence type of some subgroups of finitely generated Fuchsian groups, Ergodic Theory Dynamical Systems 1 (1981), no. 2, 209-221. MR661820 (83i:58061)

[Rob05] Thomas Roblin, Un théorème de Fatou pour les densités conformes avec applications aux revêtements galoisiens en courbure négative (French, with French summary), Israel J. Math. 147 (2005), 333-357, DOI 10.1007/BF02785371. MR2166367 (2006i:37065)

[Roc70] R. Tyrrell Rockafellar, Convex analysis, Princeton Mathematical Series, No. 28, Princeton University Press, Princeton, N.J., 1970. MR0274683 (43 \#445)

[RU08] Mario Roy and Mariusz Urbański, Real analyticity of Hausdorff dimension for higher dimensional hyperbolic graph directed Markov systems, Math. Z. 260 (2008), no. 1, 153-175, DOI 10.1007/s00209-007-0267-4. MR2413348(2009m:37064)

[Rue69] David Ruelle, Statistical mechanics: Rigorous results, W. A. Benjamin, Inc., New York-Amsterdam, 1969. MR0289084 (44 \#6279)

[Rue78] David Ruelle, Thermodynamic formalism, Encyclopedia of Mathematics and its Applications, vol. 5, Addison-Wesley Publishing Co., Reading, Mass., 1978. The mathematical structures of classical equilibrium statistical mechanics; With a foreword by Giovanni Gallavotti and Gian-Carlo Rota. MR511655 (80g:82017)

[Sar99] Omri M. Sarig, Thermodynamic formalism for countable Markov shifts, Ergodic Theory Dynam. Systems 19 (1999), no. 6, 1565-1593, DOI 10.1017/S0143385799146820. MR.1738951 (2000m:37009)

[Sar01] Omri M. Sarig, Thermodynamic formalism for null recurrent potentials, Israel J. Math. 121 (2001), 285-311, DOI 10.1007/BF02802508. MR1818392(2001m:37059)

[Sar03] Omri Sarig, Existence of Gibbs measures for countable Markov shifts, Proc. Amer. Math. Soc. 131 (2003), no. 6, 1751-1758 (electronic), DOI 10.1090/S0002-9939-0306927-2. MR 1955261 (2004b:37056)

[Sch99] Jörg Schmeling, On the completeness of multifractal spectra, Ergodic Theory Dynam. Systems 19 (1999), no. 6, 1595-1616, DOI 10.1017/S0143385799151988. MR 1738952 (2000k:37009)

[Ser81] Caroline Series, The infinite word problem and limit sets in Fuchsian groups, Ergodic Theory Dynamical Systems 1 (1981), no. 3, 337-360 (1982). MR662473 (84d:30084) 
[Sta13] Manuel Stadlbauer, An extension of Kesten's criterion for amenability to topological Markov chains, Adv. Math. 235 (2013), 450-468, DOI 10.1016/j.aim.2012.12.004. MR.3010065

[Str06] Bernd O. Stratmann, Fractal geometry on hyperbolic manifolds, Non-Euclidean geometries, Math. Appl. (N. Y.), vol. 581, Springer, New York, 2006, pp. 227-247, DOI 10.1007/0-387-29555-0_12. MR2191250 (2006g:37038)

Department of Mathematics, Graduate School of Science, Osaka University, 1-1 MachiKaneyama, Toyonaka, Osaka, 560-0043 Japan

E-mail address: jaerisch@cr.math.sci.osaka-u.ac.jp 